\title{
ENTANGLEMENT THRESHOLDS FOR RANDOM INDUCED STATES
}

\author{
GUILLAUME AUBRUN, STANISŁAW J. SZAREK, AND DEPING YE
}

\begin{abstract}
For a random quantum state on $\mathcal{H}=\mathbf{C}^{d} \otimes \mathbf{C}^{d}$ obtained by partial tracing a random pure state on $\mathcal{H} \otimes \mathbf{C}^{s}$, we consider the question whether it is typically separable or typically entangled. For this problem, we show the existence of a sharp threshold $s_{0}=s_{0}(d)$ of order roughly $d^{3}$. More precisely, for any $\varepsilon>0$ and for $d$ large enough, such a random state is entangled with very large probability when $s \leqslant(1-\varepsilon) s_{0}$, and separable with very large probability when $s \geqslant(1+\varepsilon) s_{0}$. One consequence of this result is as follows: for a system of $N$ identical particles in a random pure state, there is a threshold $k_{0}=k_{0}(N) \sim N / 5$ such that two subsystems of $k$ particles each typically share entanglement if $k>k_{0}$, and typically do not share entanglement if $k<k_{0}$. Our methods work also for multipartite systems and for "unbalanced" systems such as $\mathbf{C}^{d_{1}} \otimes \mathbf{C}^{d_{2}}, d_{1} \neq d_{2}$. The arguments rely on random matrices, classical convexity, high-dimensional probability and geometry of Banach spaces; some of the auxiliary results may be of reference value.
\end{abstract}

\section{INTRODUCTION}

In recent years, random constructions have become a very fruitful tool in quantum information theory. The study of random channels and random states has particularly intensified since the influential paper 22. The most successful achievement of the probabilistic method in quantum information theory is arguably Hastings's proof that suitably chosen random channels provide a counterexample to the additivity conjecture for classical capacity of quantum channels [21].

In this paper, we address the most fundamental question one can ask about a random state: is it entangled? Detecting and exploiting entanglement, first discovered in the 1930's [14, is a central problem in quantum information and quantum computation at least since Shor's work [44] on integer factoring. However, the structure of the set of entangled quantum states is still not well-understood. The well-known Peres-Horodecki positive partial transpose (PPT) criterion [24, 37] is a necessary condition for (lack of) entanglement, but this condition is sufficient only for qubit-qubit and qubit-qutrit systems [45, 52].

We consider here a family of random states that are known as random induced states. These are mixed states on $\mathcal{H}$ obtained after partial tracing, over some ancilla space $\mathcal{H}_{a}$, a uniformly distributed pure state on $\mathcal{H} \otimes \mathcal{H}_{a}$. This leads to a natural family of probability measures on the set of states on $\mathcal{H}$ (see [56, 9]), where $\operatorname{dim} \mathcal{H}_{a}$, the dimension of the environment, is a parameter. Indeed, if all that we know about the system $\mathcal{H} \otimes \mathcal{H}_{a}$ are the dimensions of the factors $\mathcal{H}$ and $\mathcal{H}_{a}$, and that it is isolated from the rest of the environment, the corresponding random induced state is a reasonable model for, or at least a reasonable first guess about the state of the system $\mathcal{H}$.

Of course, the induced state $\rho$ being random, we cannot expect to be able to tell what $\rho$ is. However, we may be able to infer some properties of $\rho$ if they are generic (that is, occur with probability close to 1) for a given random model. For specificity, consider $\mathcal{H}=\mathbf{C}^{d} \otimes \mathbf{C}^{d}$ and let us focus on the question "Is a random state entangled?" As it turns out, the answer depends in a crucial and rather precise way on

Key words and phrases. Entanglement, quantum states, random quantum states. 
the size of the environment with low-dimensional environments leading typically to entangled states and high-dimensional environments leading to separable states.

In the special case $\operatorname{dim} \mathcal{H}_{a}=\operatorname{dim} \mathcal{H}=d^{2}$, we are led to the uniform distribution on the set of states (i.e., uniform with respect to the usual Hilbert-Schmidt volume). As was shown in [4], the proportion of states (again, measured with respect to the Hilbert-Schmidt volume) that are separable is extremely small in large dimensions. This means that when $\operatorname{dim} \mathcal{H}_{a}=d^{2}$, random induced states are typically entangled. This was extended to the case when $\operatorname{dim} \mathcal{H}_{a}$ is slightly larger than $d^{2}$ in [54, 3].

On the other hand, it was proved in $\left[22\right.$ that random induced states on $\mathbf{C}^{d} \otimes \mathbf{C}^{d}$ are typically separable when $\operatorname{dim} \mathcal{H}_{a}$ is proportional to $d^{4}$. In the present paper, we bridge the gap between these estimates and show that the threshold $s_{0}(d)$ between separability and entanglement occurs at order $d^{3}$ (more precisely, we obtain the inequalities $c d^{3} \leqslant s_{0}(d) \leqslant C d^{3} \log ^{2} d$, where $C, c>0$ are universal constants, independent of the dimensions involved). More specifically, we show that for any $\varepsilon>0$, the following holds if $d$ is large enough. When the environment dimension $s=\operatorname{dim} \mathcal{H}_{a}$ is smaller than $(1-\varepsilon) s_{0}$, the random induced state is entangled with overwhelming probability. When the environment dimension exceeds $(1+\varepsilon) s_{0}$, the random induced state is separable with overwhelming probability.

The heuristics behind the consequence stated in the abstract are now as follows. If we have a system of $N$ particles (with $D$ levels each) which is in a random pure state, and two subsystems of $k$ particles each, then the "joint state" of the subsystems is modeled by a random induced state on $\mathbf{C}^{d} \otimes \mathbf{C}^{d}$ with $d=D^{k}$ and $s=D^{N-2 k}$. In particular, the relation $k=N / 5$, or $N=5 k$, corresponds exactly to $s=d^{3}$. The reason for the threshold effect is that passing from $k$ to $k-1$ increases $s$ by a factor of $D^{2} \geqslant 4$, while - as stated above - the transition from generic entanglement to generic separability takes place when $s$ is increased only slightly (the simultaneous decrease of $d$ by a factor of $D \geqslant 2$ only amplifies the effect).

Our method of proof is geometric and uses tools from high-dimensional convexity, a field also known as asymptotic geometric analysis. This has become a fruitful approach to study the geometry of quantum states in large dimension; recent contributions include, for instance, [5, 49, 15. Asymptotic geometric analysis strives to understand properties of geometric structures in high dimension, which - because of the "central limit theorem-like" effects - are believed to be relatively easier to pinpoint than those in low dimension. The phenomenon of concentration of measure plays a central role in the whole theory.

While our starting point is an estimation of the Hilbert-Schmidt volume of the set $\mathcal{S}$ of separable states on $\mathbf{C}^{d} \otimes \mathbf{C}^{d}$, the relevant geometric parameter is not the volume itself, but rather the mean width of the dual set $\mathcal{S}^{\circ}$. We estimate it in an indirect way, using the $M M^{*}$-estimate, a general result from asymptotic geometric analysis.

Our method also applies to the case of multipartite systems $\left(\mathbf{C}^{d}\right)^{\otimes k}$. In the asymptotic limit, when $k$ is fixed and $d$ goes to infinity, we show that the threshold for separability vs. entanglement occurs for $s$ of order $d^{2 k-1}$ (up to logarithmic factors). However, to keep the exposition as simple as possible, we focus on the bipartite case $k=2$ in most of the paper.

Finally, we also show that "one half" of our main result (entanglement is generic for small $\operatorname{dim} \mathcal{H}_{a}$ ) can be proved in a more elementary way by working with the densities of induced measures. Similar arguments appeared already in the papers [53, 54].

This paper is organized as follows. In Section 2, we introduce some mathematical background, necessary notation, state our main theorem and present a high-level overview of the proof. A rigorous proof is found in Sections 3 and 4 . To not to obscure the structure of the proof, some general (but technically involved) mathematical tools are collected and/or developed in several Appendices, some of which may be of independent interest and/or reference value. In Section 5 , we extend our results to the case of 
multipartite systems. In Section 6, we will provide another, more elementary, proof of our result, which is valid only in the regime where entanglement is generic. Miscellaneous remarks and loose ends are addressed in Section 7 .

General references for concepts related to quantum information theory are [9, 36], for those related to asymptotic geometric analysis [34, 38, 28, and for those related to random matrices [1, 13. A high-level non-technical overview of the results of this paper and of a related article [3] can be found in [6].

\section{Notation, BaCkground And the Statement of the main theorem}

The letters $C, c, c_{0}, \ldots$ denote absolute numerical constants (independent of the instance of the problem) whose values may change from place to place. When $A, B$ are quantities depending on the dimension (and perhaps some other parameters), the notation $A \lesssim B$ means that there exists an absolute constant $C>0$ such that the inequality $A \leqslant C B$ holds in every dimension. Similarly $A \simeq B$ means both $A \lesssim B$ and $B \lesssim A$. As usual, $A \sim B$ means that $A / B \rightarrow 1$ as the dimension (or some other relevant parameter) tends to $\infty$, while $A=o(B)$ means that $A / B \rightarrow 0$.

There are various dimensions appearing repeatedly in this paper, and we will stick to the following notational scheme. We will work in a complex Hilbert space $\mathcal{H}=\mathbf{C}^{n}$, with $n=d^{k}$, so that we may identify $\mathbf{C}^{n}$ with $\left(\mathbf{C}^{d}\right)^{\otimes k}$ ( $k=2$ in most of the paper). We will always assume that $d \geqslant 2$. The set of states on $\mathbf{C}^{n}$ has real dimension $m=n^{2}-1$. We also consider an ancilla space $\mathbf{C}^{s}$, and pure states in $\mathbf{C}^{n} \otimes \mathbf{C}^{s}$. Occasionally the unit sphere in $\mathbf{C}^{n} \otimes \mathbf{C}^{s}$ will be identified with the unit sphere $S^{m-1} \subset \mathbf{R}^{m}$, with $m=2 n s$. General results from convex geometry will also be stated in $\mathbf{R}^{m}$ (except in Appendices $\mathrm{A}$ and $\mathrm{B}$, where we consider $\mathbf{R}^{\operatorname{dim} \mathcal{H}}$, and so $\mathbf{R}^{n}$ is more appropriate).

2.1. Basic facts from convex geometry. A convex body $K$ in a real finite-dimensional vector space (usually identified with $\mathbf{R}^{m}$ ) is a convex compact set with non-empty interior. We denote by $|\cdot|$ the Euclidean norm. A convex body $K$ is symmetric if $K=-K$. The convex bodies we consider may be nonsymmetric, but we usually "arrange" that the origin belongs to the interior of $K$. The gauge associated to such $K$ is the function $\|\cdot\|_{K}$ defined for $x \in \mathbf{R}^{m}$ by

$$
\|x\|_{K}:=\inf \{t \geqslant 0: x \in t K\} .
$$

If $K$ is symmetric, $\|\cdot\|_{K}$ is a norm and $K$ is precisely the unit ball in that norm. However, in general, we may have $\|x\|_{K} \neq\|-x\|_{K}$ when $K$ is non-symmetric.

By vol we denote the Lebesgue measure on $\mathbf{R}^{m}$. The volume radius of a convex body $K \subset \mathbf{R}^{m}$ is defined as

$$
\operatorname{vrad}(K):=\left(\frac{\operatorname{vol} K}{\operatorname{vol} B_{2}^{m}}\right)^{1 / m}
$$

where $B_{2}^{m}$ denotes the unit Euclidean ball. In words, $\operatorname{vrad}(K)$ is the radius of the Euclidean ball with same volume as $K$. The same notation will be used if $K$ "lives" in an $m$-dimensional linear or affine subspace of a larger space.

If $K \subset \mathbf{R}^{m}$ is a convex body with origin in the interior, the polar of $K$ is the convex body $K^{\circ}$ defined as

$$
K^{\circ}:=\left\{y \in \mathbf{R}^{m}:\langle x, y\rangle \leqslant 1 \text { for all } x \in K\right\} .
$$

A basic result from convex analysis is that $\left(K^{\circ}\right)^{\circ}=K$ (this is the bipolar theorem, a baby version of the Hahn-Banach theorem).

The inradius of a convex body $K$ is the largest radius $r$ of a Euclidean ball contained in $K$. Similarly, the outradius of $K$ is the smallest $R>0$ such that $K$ is contained in a ball of radius $R$. In most cases 
the optimal balls will be centered at the origin, then $r$ and $R$ can be equivalently defined as the "best" constants for which $R^{-1}|\cdot| \leqslant\|\cdot\|_{K} \leqslant r^{-1}|\cdot|$.

If $u$ is a vector from the unit sphere $S^{m-1}$, the support function of $K$ in the direction $u$ is $h_{K}(u):=$ $\max _{x \in K}\langle x, u\rangle=\|u\|_{K^{\circ}}$. Note that $h_{K}(u)$ is the distance from the origin to the hyperplane tangent to $K$ in the direction $u$. The mean width 1 of $K$ is then defined as

$$
w(K):=\int_{S^{m-1}} h_{K}(u) d \sigma(u)=\int_{S^{m-1}}\|u\|_{K^{\circ}} d \sigma(u),
$$

where $d \sigma(u)$ is the normalized spherical measure on the sphere $S^{m-1}$ (this definition makes sense for any bounded set $K)$.

The Urysohn's inequality (see, e.g., [38]) is a fundamental result which compares the volume radius and the mean width: for any convex body $K \subset \mathbf{R}^{m}$, we have

$$
\operatorname{vrad}(K) \leqslant w(K) .
$$

It is often convenient to consider the Gaussian variant of mean width

$$
w_{G}(K):=\mathbf{E}\|G\|_{K^{\circ}},
$$

where $G$ is a standard Gaussian vector in $\mathbf{R}^{m}$, i.e., a random vector with independent $N(0,1)$ coordinates in any orthonormal basis. One checks, by passing to polar coordinates, that for every convex body $K \subset \mathbf{R}^{m}$

$$
w_{G}(K)=\gamma_{m} w(K)
$$

where

$$
\gamma_{m}:=\mathbf{E}|G|=\frac{\sqrt{2} \Gamma((m+1) / 2)}{\Gamma(m / 2)}, \quad \sqrt{m-1} \leqslant \gamma_{m} \leqslant \sqrt{m}
$$

is a constant depending only on $m$.

Note that we may extend the definition of the Gaussian mean width to all bounded sets $K \subset \mathbf{R}^{m}$ through the formula

$$
w_{G}(K)=\mathbf{E} \sup _{x \in K}\langle G, x\rangle .
$$

The Gaussian mean width of $K$ is an intrinsic parameter and does not depend on the ambient dimension: if $K$ lives in a subspace $E \subset \mathbf{R}^{m}$, the formula (5) gives the same value whether $G$ is a standard Gaussian vector in $\mathbf{R}^{m}$, or a standard Gaussian vector in $E$.

2.2. Concentration of measure. The phenomenon of concentration of measure plays a central role in our proofs. We first recall the statement of Lévy's lemma. In the statements of Lemmas 2.1 and 2.2 , $\mathbf{P}$ stands for the uniform measure on the sphere $S^{m-1}$, normalized so that $\mathbf{P}\left(S^{m-1}\right)=1$.

Lemma 2.1 (Lévy's lemma [29, 34]). If $f: S^{m-1} \rightarrow \mathbf{R}$ is an L-Lipschitz function, then for every $\varepsilon>0$,

$$
\mathbf{P}(\{|f-M|>\varepsilon\}) \leqslant C_{1} \exp \left(-c_{1} m \varepsilon^{2} / L^{2}\right),
$$

where $M$ is any central value of $f$, and $C_{1}, c_{1}>0$ are absolute constants.

\footnotetext{
${ }^{1}$ It would have been geometrically more precise to call this quantity the mean half-width.
} 
By a central value of a random variable $X$ we mean either the expectation or the median, or more generally any number $M$ such that $\mathbf{P}(X \geqslant M) \geqslant 1 / 4$ and $\mathbf{P}(X \leqslant M) \geqslant 1 / 4$. Any two central values for the function $f$ appearing in Lévy's lemma differ by at most $C_{2} L / \sqrt{m}$.

While we will be mostly interested in concentration of functions on the sphere, the phenomenon appears also in many other contexts, for example in the Gaussian setting. A recent fairly comprehensive reference is the monograph 28$]$.

We will also consider situations in which a function $f$ has a (possibly) large Lipschitz constant, while the restriction of $f$ to a large subset has a small Lipschitz constant. The following extension of Lévy's lemma handles such a case. The trick behind this lemma appeared in [5] and implicitly in [21].

Lemma 2.2 (Lévy's lemma, local version). Let $\Omega \subset S^{m-1}$ be a subset of measure larger than 3/4. Let $f: S^{m-1} \rightarrow \mathbf{R}$ be a function such that the restriction of $f$ to $\Omega$ is L-Lipschitz. Then, for every $\varepsilon>0$,

$$
\mathbf{P}\left(\left\{\left|f(x)-M_{f}\right|>\varepsilon\right\}\right) \leqslant \mathbf{P}\left(S^{m-1} \backslash \Omega\right)+C_{1} \exp \left(-c_{1} m \varepsilon^{2} / L^{2}\right),
$$

where $M_{f}$ is the median of $f$, and $C_{1}, c_{1}>0$ are absolute constants.

In Lemma 2.2, the median can be replaced by another quantile (up to changes in the numerical constants). However, in general, it cannot be replaced by the mean (we do not assume any regularity of $f$ outside $\Omega$, therefore the expectation may even fail to be well-defined). Still, more often than not, some information about global regularity of $f$ is available and concentration around the mean can be inferred; see the comment at the end of the proof and the remark following Lemma 3.4 below.

Proof of Lemma 2.2. The key point is that in any metric space $X$, it is possible to extend any $L$-Lipschitz function $h$ defined on a subset $Y$ without increasing the Lipschitz constant. Use, e.g., the formula

$$
\tilde{h}(x)=\inf _{y \in Y}[h(y)+L \operatorname{dist}(x, y)] .
$$

By applying this fact to $X=S^{m-1}, Y=\Omega$ and $h=f_{\mid \Omega}$, we obtain a function $\tilde{f}: S^{m-1} \rightarrow \mathbf{R}$ which is $L$-Lipschitz and coincides with $f$ on $\Omega$. Moreover, if $M=M_{f}$ is the median of $f$, then

$$
\mathbf{P}(\{\tilde{f} \geqslant M\}) \geqslant \mathbf{P}(\Omega \cap\{\tilde{f} \geqslant M\})=\mathbf{P}(\Omega \cap\{f \geqslant M\}) \geqslant 1 / 4 .
$$

Similarly, $\mathbf{P}(\{\tilde{f} \leqslant M\}) \leqslant 1 / 4$. Hence $M$ is a central value for $\tilde{f}$. By Lévy's lemma,

$$
\mathbf{P}(\{|\tilde{f}-M|>\varepsilon\}) \leqslant C_{1} \exp \left(-c_{1} m \varepsilon^{2} / L^{2}\right) .
$$

Therefore,

$$
\begin{aligned}
\mathbf{P}(\{|f-M|>\varepsilon\}) & \leqslant \mathbf{P}(\{f \neq \tilde{f}\})+\mathbf{P}(\{|\tilde{f}-M|>\varepsilon\}) \\
& \leqslant \mathbf{P}\left(S^{m-1} \backslash \Omega\right)+C_{1} \exp \left(-c_{1} m \varepsilon^{2} / L^{2}\right),
\end{aligned}
$$

as claimed. Note that if we know, for example, that $f$ is "reasonably bounded," then we can infer that the median $M$ and the mean $\mathbf{E} f$ of $f$ do not differ very much (e.g., $\left.|M-\mathbf{E} f| \lesssim\|f-M\|_{\infty} \mathbf{P}\left(S^{m-1} \backslash \Omega\right)\right)$ and deduce a posteriori concentration around the mean.

2.3. Quantum states. Throughout the paper, we consider a (finite dimensional) complex Hilbert space $\mathcal{H}$, equipped with a norm which we will also denote by $|\cdot|$.

A quantum state on $\mathcal{H}$ is a positive trace one operator on $\mathcal{H}$. We use $\mathcal{D}=\mathcal{D}(\mathcal{H})$ to denote the set of all quantum states on $\mathcal{H}$. The extreme points of this set are pure states, in particular if $\mathcal{H}=\mathbf{C}^{n}$, then

$$
\mathcal{D}\left(\mathbf{C}^{n}\right)=\operatorname{conv}\left\{|\psi\rangle\left\langle\psi\left|: \psi \in \mathbf{C}^{n},\right| \psi\right|=1\right\} .
$$


Above (and, when convenient, in what follows) we use Dirac's bra-ket notation: $|\psi\rangle$ is a column vector, $\langle\psi|=| \psi\rangle^{\dagger}$ is a row vector and $|\psi\rangle\langle\psi|$ is their outer product, the orthogonal projection onto $\mathbf{C} \psi$. The set $\mathcal{D}\left(\mathbf{C}^{n}\right)$ is contained in the (real) space $\mathcal{M}_{n}^{s a}$ of $n \times n$ self-adjoint matrices, endowed with the HilbertSchmidt inner product $\langle A, B\rangle=\operatorname{tr}(A B)$. Whenever considering a geometric invariant (inradius, mean width, ... ) of a set of matrices, it will be tacitly understood that the Hilbert-Schmidt Euclidean structure is used. This applies also to spaces of not-necessarily-self-adjoint and/or rectangular matrices; in that case $\langle A, B\rangle=\operatorname{tr}\left(A B^{\dagger}\right)$. We will also occasionally use the Schatten $p$-norm $\|A\|_{p}=\left(\operatorname{tr}\left(A^{\dagger} A\right)^{p / 2}\right)^{1 / p}$. The limit case $\|\cdot\|_{\infty}$ coincides with the operator norm $\|\cdot\|_{o p}$ (from the category of normed spaces), while $\|\cdot\|_{2}=\|\cdot\|_{H S}$ is the Hilbert-Schmidt (or Frobenius) norm.

For every dimension $n$, we introduce now a family of probability distributions on $\mathcal{D}\left(\mathbf{C}^{n}\right)$ which plays a central role in this paper. These probability measures are known as induced measures and can be described as follows. Fix a positive integer $s$ and let $|\psi\rangle\langle\psi|$ be a random pure state on the Hilbert space $\mathbf{C}^{n} \otimes \mathbf{C}^{s}$, where $\psi$ is a random unit vector uniformly distributed on the sphere in $\mathbf{C}^{n} \otimes \mathbf{C}^{s}$. Then consider the partial trace of $|\psi\rangle\langle\psi|$ over $\mathbf{C}^{s}$; the resulting state is a random state on $\mathbf{C}^{n}$ and we denote by $\mu_{n, s}$ its distribution.

When $s \geqslant n$, the probability measure $\mu_{n, s}$ has a density with respect to the Lebesgue measure on $\mathcal{D}\left(\mathbf{C}^{n}\right)$ which has a simple form [56]

$$
\frac{d \mu_{n, s}}{d \operatorname{vol}}(\rho)=\frac{1}{Z_{n, s}}(\operatorname{det} \rho)^{s-n},
$$

where $Z_{n, s}$ is a normalization factor. Note that formula (6) allows to define the measure $\mu_{n, s}$ (in particular) for every real $s \geqslant n$, while the partial trace construction makes sense only for integer values of $s$.

In the important special case when $s=n$, the density of the measure $\mu_{n, n}$ is constant. A random state distributed according to $\mu_{n, n}$ is uniformly distributed on $\mathcal{D}\left(\mathbf{C}^{n}\right)$ (i.e., uniformly with respect to the Lebesgue measure).

It is important to consider the case when the Hilbert space $\mathcal{H}$ itself carries a tensor product structure. For simplicity we will largely focus on the bipartite balanced case where $\mathcal{H}=\mathbf{C}^{d} \otimes \mathbf{C}^{d}$ (multipartite Hilbert spaces are considered in Sections 5 and 6, while some remarks on extensions to the unbalanced setting $\mathcal{H}=\mathbf{C}^{d_{1}} \otimes \mathbf{C}^{d_{2}}, d_{1} \neq d_{2}$ are given in Section (7.2).

A quantum state on $\mathbf{C}^{d} \otimes \mathbf{C}^{d}$ can be either separable or entangled, and this dichotomy is fundamental in quantum theory. By definition [51, a state $\rho$ is separable if it can be written as a convex combination of product states (i.e. states of the form $\rho_{1} \otimes \rho_{2}$, where $\rho_{1}, \rho_{2}$ are states on $\mathbf{C}^{d}$ ). If we denote by $\mathcal{S}\left(\mathbf{C}^{d} \otimes \mathbf{C}^{d}\right)$ $\subset \mathcal{D}\left(\mathbf{C}^{d} \otimes \mathbf{C}^{d}\right)$ the subset of separable states, an equivalent description is the following

$$
\mathcal{S}\left(\mathbf{C}^{d} \otimes \mathbf{C}^{d}\right)=\operatorname{conv}\left\{\left|\psi_{1} \otimes \psi_{2}\right\rangle\left\langle\psi_{1} \otimes \psi_{2}\left|: \psi_{1}, \psi_{2} \in \mathbf{C}^{d},\right| \psi_{1}|=| \psi_{2}\right|=1\right\}
$$

A state which is not separable is called entangled. We denote $n=d^{2}$ the (complex) dimension of the space $\mathbf{C}^{d} \otimes \mathbf{C}^{d}$, which we identify with $\mathbf{C}^{n}$. The affine hyperplane

$$
\mathcal{M}_{n}^{s a, 1}=\left\{A \in \mathcal{M}_{n}^{s a}: \operatorname{tr}(A)=1\right\},
$$

contains the set $\mathcal{D}=\mathcal{D}\left(\mathbf{C}^{n}\right)$ of states and its subset $\mathcal{S}=\mathcal{S}\left(\mathbf{C}^{n}\right)$ of separable states; they are both of full (real) dimension $m=n^{2}-1$.

We want to consider $\mathcal{M}_{n}^{s a, 1}$ as a vector space where the role of the origin is played by the maximally mixed state $\mathbb{I} / n$, where $\mathbb{I}$ denotes the identity matrix. One way to formalize this point of view is to work with the linear hyperplane

$$
\mathcal{M}_{n}^{s a, 0}=\left\{A \in \mathcal{M}_{n}^{s a} \quad: \quad \operatorname{tr}(A)=0\right\}=\mathcal{M}_{n}^{s a, 1}-\mathbb{I} / n
$$


and with the translated convex body

$$
\mathcal{S}_{0}=\mathcal{S}-\mathbb{I} / n=\{\rho-\mathbb{I} / n: \rho \in \mathcal{S}\} \subset \mathcal{M}_{n}^{s a, 0} .
$$

Similarly, we denote $\mathcal{D}_{0}=\mathcal{D}-\mathbb{I} / n$ (in the sequel, we will use analogous notation also for other sets). The geometry of the sets $\mathcal{S}$ and $\mathcal{D}$ plays a central role in our argument. Estimates on some known geometric parameters associated to these convex bodies are gathered in Table 1.

TABLE 1. Radii of $\mathcal{D}$ and $\mathcal{S}$ for $\mathbf{C}^{d} \otimes \mathbf{C}^{d}$, where $n=d^{2}$. All these parameters are translation invariant, so their values for $\mathcal{D}_{0}$ and $\mathcal{S}_{0}$ are respectively the same. In each row the quantities increase from left to right.

\begin{tabular}{|c|c|c|c|c|}
\hline & inradius & volume radius & mean width & outradius \\
\hline $\mathcal{D}\left(\mathbf{C}^{d} \otimes \mathbf{C}^{d}\right)$ & $=1 / \sqrt{n(n-1)}$ & $\simeq n^{-1 / 2}$ & $\simeq n^{-1 / 2}$ & $=\sqrt{(n-1) / n}$ \\
\hline $\mathcal{S}\left(\mathbf{C}^{d} \otimes \mathbf{C}^{d}\right)$ & $=1 / \sqrt{n(n-1)}$ & $\simeq n^{-3 / 4}$ & $\simeq n^{-3 / 4}$ & $=\sqrt{(n-1) / n}$ \\
\hline
\end{tabular}

The volume of $\mathcal{D}$ was computed exactly in [55] and it was noted in [46] that the mean width and the volume radius have the same order. The remarkable fact that $\mathcal{D}$ and $\mathcal{S}$ have the same inradius was proved in [18. (An alternative argument is based on a dual formulation given in [48, a proof of which was provided by H.-J. Sommers, see [35.) Sharp bounds on the volume radius of $\mathcal{S}$ were given in [4] (the $\operatorname{ratio} \operatorname{vrad}(\mathcal{S}) / \operatorname{vrad}(\mathcal{D})$ is estimated in Theorem 1 in $[4]$. The estimate for the mean width of $\mathcal{S}$ does not appear explicitly in [4], but follows from the argument since the upper bound on the volume radius was obtained via Urysohn's inequality (2). Finally, the calculation of the outradii is easy: they are attained on pure states. Note that the inradii and the outradii of $\mathcal{D}$ and $\mathcal{S}$ are attained on balls centered at $\mathbb{I} / n$, which is the only point invariant under isometries of each of these bodies.

It is easily checked that $\mathcal{D}_{0}\left(\mathbf{C}^{n}\right)^{\circ}=-n \mathcal{D}_{0}\left(\mathbf{C}^{n}\right)$ (we recall that the polar operation ${ }^{\circ}$ is performed in the space $\mathcal{M}_{n}^{s a, 0}$ of trace zero matrices). This is a consequence of the fact that the cone of positive matrices is self-dual (cf. more general comments in the second paragraph of Section 4). We deduce immediately from Table 1 that the mean width of $\mathcal{D}_{0}\left(\mathbf{C}^{n}\right)^{\circ}$ is of order $\sqrt{n}$. The situation is not so simple for $\mathcal{S}$, and estimating the mean width of $\mathcal{S}_{0}\left(\mathbf{C}^{d} \otimes \mathbf{C}^{d}\right)^{\circ}$ is the main technical difficulty in our argument.

2.4. Threshold for entanglement vs separability. The main result of the paper is the following theorem.

Theorem 2.3. There are effectively computable absolute constants $C, c>0$ and a function $s_{0}(d)$ satisfying

$$
c d^{3} \leqslant s_{0}(d) \leqslant C d^{3} \log ^{2} d
$$

such that if $\rho$ is a random state on $\mathbf{C}^{d} \otimes \mathbf{C}^{d}$ distributed according to the measure $\mu_{d^{2}, s}$, then, for any $\varepsilon>0$,

(i) if $s \leqslant(1-\varepsilon) s_{0}(d)$, we have

$$
\mathbf{P}(\rho \text { is separable }) \leqslant 2 \exp \left(-c(\varepsilon) d^{3}\right),
$$

(ii) if $s \geqslant(1+\varepsilon) s_{0}(d)$ we have

$$
\mathbf{P}(\rho \text { is entangled }) \leqslant 2 \exp (-c(\varepsilon) s),
$$

where $c(\varepsilon)$ is a positive constant depending only on $\varepsilon$. 
Theorem 2.3 asserts that, for fixed $d$, the character of the induced state changes sharply from generic entanglement to generic separability as $s$, the dimension of the ancilla, increases. If we knew that the threshold function $s_{0}(\cdot)$ was regular enough, an analogous statement with the roles of $d$ and $s$ exchanged would immediately follow. The following is a result in that direction which can be deduced with relatively little effort. (A statement in a similar language - but much less precise - appears in [26].)

Corollary 2.4. Consider a system of $N$ identical particles (qudits) in a random pure state. Then there is a threshold $k_{0}=k_{0}(N) \sim N / 5$ such that two subsystems of $k$ particles each typically share entanglement if $k>k_{0}$, and typically do not share entanglement if $k<k_{0}$.

We next describe the threshold function $s_{0}(d)$ appearing in the main theorem. Let $G$ denote the standard Gaussian vector in the space $\mathcal{M}_{n}^{s a, 0}$, which we will also call a GUE ${ }^{0}$ random matrix. One may represent $G$ as follows. Start from an $n \times n$ GUE random matrix $G^{\prime}$, which is the standard Gaussian vector in the space $\mathcal{M}_{n}^{\text {sa }}$ (see [1, 13]). Then $G$ can be realized as $G^{\prime}-\frac{\operatorname{tr} G^{\prime}}{n} \mathbb{I}$ (a conditional expectation of $G^{\prime}$ ). Equivalently, one may realize $G$ by conditioning $G^{\prime}$ to be of trace 0 . In this notation, $s_{0}$ is defined as follows.

Definition 2.5. For every integer $d$, we define $s_{0}=s_{0}(d)$ by the formula

$$
s_{0}(d):=\left(\frac{\mathbf{E}\|G\|_{\mathcal{S}_{0}}}{d^{2}}\right)^{2} \sim w\left(\mathcal{S}_{0}^{\circ}\right)^{2},
$$

where $G$ is a $\mathrm{GUE}^{0}$ matrix of size $d^{2} \times d^{2}$. (The relation " " is justified by (3) and (4).)

2.5. Overview of the proof. Our proof of Theorem 2.3 consists of two largely independent parts

- showing that $s_{0}$ defined by (77) is indeed a sharp threshold for the separability of random states, - proving that $d^{3} \lesssim s_{0} \lesssim d^{3} \log ^{2} d$.

The details of the two parts will be dealt with in Sections 3 and 4 respectively (except for some fine points regarding the probability estimate in part (i) of the Theorem, which are clarified in Section 7.5). To not to obscure the structure of the proof, some general (but technically involved) auxiliary results are relegated to appendices. The heuristic behind deducing Corollary 2.4 from Theorem 2.3 was explained in the Introduction. A rigorous argument requires two additional simple observations (Lemmas 3.6 and 7.3 ) and is sketched in Section 7.6 .

And here is a "high level" overview of the argument. We first note tautological equivalences

$\rho$ is separable $\Longleftrightarrow \rho \in \mathcal{S} \Longleftrightarrow \rho-\mathbb{I} / n \in \mathcal{S}_{0} \Longleftrightarrow\|\rho-\mathbb{I} / n\|_{\mathcal{S}_{0}} \leqslant 1$.

This means that Theorem 2.3 asserts that if $s$ is noticeably larger than $s_{0}$ (that is, if $\left.s \geqslant(1+\varepsilon) s_{0}\right)$, then $\|\rho-\mathbb{I} / n\|_{\mathcal{S}_{0}} \leqslant 1$ with probability close to 1 , and if $s$ is noticeably smaller than $s_{0}$, then $\|\rho-\mathbb{I} / n\|_{\mathcal{S}_{0}}>1$ with probability close to 1 .

The strategy is now to show that the function $\rho \rightarrow\|\rho-\mathbb{I} / n\|_{\mathcal{S}_{0}}$ is sufficiently regular (which is relatively straightforward) and that whenever $s$ is noticeably larger than $s_{0}$, then the median (or the expected value) of $\|\rho-\mathbb{I} / n\|_{\mathcal{S}_{0}}$ is noticeably smaller than 1. The concentration phenomenon (Lemmas 2.1 and 2.2) then implies that $\|\rho-\mathbb{I} / n\|_{\mathcal{S}_{0}} \leqslant 1$ with probability close to 1 , as needed. Similarly, if $s$ is noticeably smaller than $s_{0}$, we need to establish that the expected value of $\|\rho-\mathbb{I} / n\|_{\mathcal{S}_{0}}$ is noticeably larger than 1.

The problem thus reduces to figuring out the dependence of the expected value of $\|\rho-\mathbb{I} / n\|_{\mathcal{S}_{0}}$ on the ancilla dimension $s$, which is implicit in the definition of the random state $\rho=\rho_{n, s}=\operatorname{tr}_{\mathbf{C}^{s}}|\psi\rangle\langle\psi|$. This turns out to be not so easy, partly because of the non-linear dependence of $\rho$ on $\psi$ (a random vector uniformly distributed on the unit sphere in $\mathbf{C}^{n} \otimes \mathbf{C}^{s}$ ). However, it turns out (see Proposition [3.1] below) 
that, for all practical purposes, $\rho_{n, s}-\mathbb{I} / n$ is equivalent to $A_{n, s}:=\frac{1}{n \sqrt{s}} G$, where $G$ is a random matrix distributed according to the standard Gaussian measure in the space of $n \times n$ self-adjoint matrices with vanishing trace $\left(\mathrm{GUE}^{0}\right.$, defined above in Section 2.4).

This simplifies matters significantly since, first, the dependence of $A_{n, s}$ on $s$ is very straightforward and, second, because the expected value of $\|G\|_{\mathcal{S}_{0}}$ has geometric meaning: as explained in Section 2.1, it is explicitly related to $w\left(\mathcal{S}_{0}^{\circ}\right)$, the mean width of the polar of $\mathcal{S}_{0}$. However, estimating $w\left(\mathcal{S}_{0}^{\circ}\right)$ directly is still hard. The approach which succeeds is to proceed through a duality argument, the idea being that for a "well-balanced" convex body $K$, its mean width $w(K)$ and the mean width of its polar, $w\left(K^{\circ}\right)$, are approximately reciprocal, and that good estimates for $w\left(\mathcal{S}_{0}\right)$ exist in the literature.

Some of the steps indicated above (for example, showing that the set $\mathcal{S}_{0}$ is well-balanced in the needed sense) are quite involved by themselves; we will try to convey additional heuristic arguments clarifying such steps once precise statements are formulated and once appropriate notation is available.

\section{Proof that $s_{0}$ IS A threshold For SEPARABILITy}

Let $\rho=\rho_{n, s}$ be a random state on $\mathbf{C}^{n}$ with distribution $\mu_{n, s}$. The first step is to approximate $\rho-\mathbb{I} / n$ by $\frac{1}{n \sqrt{s}} G$, where $G=G_{n}$ is an $n \times n \mathrm{GUE}^{0}$ random matrix. (This step would not be necessary if we were able to define the threshold dimension $s_{0}$ via the expected value - or median - of $\|\rho-\mathbb{I} / n\|_{\mathcal{S}_{0}}$ rather than in terms of the expected value of $\|G\|_{\mathcal{S}_{0}}$.) Here is a precise statement.

Proposition 3.1. Denote by $c_{n, s}\left(\right.$ resp. $C_{n, s}$ ) the largest (resp. smallest) constant such that for every convex body $K \subset \mathcal{M}_{n}^{\text {sa,0 }}$ containing 0 in its interior, if $\rho$ is a random state on $\mathbf{C}^{n}$ distributed according to $\mu_{n, s}$, and if $G$ is a standard Gaussian vector in $\mathcal{M}_{n}^{s a, 0}$, we have

$$
\frac{c_{n, s}}{n \sqrt{s}} \mathbf{E}\|G\|_{K} \leqslant \mathbf{E}\left\|\rho-\frac{\mathbb{I}}{n}\right\|_{K} \leqslant \frac{C_{n, s}}{n \sqrt{s}} \mathbf{E}\|G\|_{K}
$$

Then

$$
\lim _{n, \frac{s}{n} \rightarrow \infty} C_{n, s}=\lim _{n, \frac{s}{n} \rightarrow \infty} c_{n, s}=1 .
$$

For the record, let us clarify what double-indexed limits mean (here and later). The statement

$$
\lim _{n, \frac{s}{n} \rightarrow \infty} C_{n, s}=1
$$

is supposed to signify the following: for any sequences $\left(n_{k}\right),\left(s_{k}\right)$ such that both $\left(n_{k}\right)$ and $\left(s_{k} / n_{k}\right)$ tend to infinity, we have $\lim _{k \rightarrow \infty} C_{n_{k}, s_{k}}=1$.

A rigorous proof of Proposition 3.1 is given in Appendix B here we restrict ourselves to some heuristic comments. First, both $\rho=\rho_{n, s}$ and $G=G_{n}$ are well-known ensembles in Random Matrix Theory. They are both invariant under conjugation with a unitary matrix, and their asymptotic spectral properties have been thoroughly studied.

The behavior of $G_{n}$ for large $n$ is governed by the famous Wigner's semi-circle law. On the other hand, (appropriately normalized) $\rho_{n, s}$ is known as the Wishart ensemble and, when $n, s \rightarrow \infty$ with the ratio $s / n \rightarrow \beta$ for some $\beta>0$, the limiting spectral distribution is given by the Marchenko-Pastur law. However, in the asymptotic regime that is relevant here $(n, s / n \rightarrow \infty)$, the limiting spectral distribution is also a (non-centered, that's why we subtract $\mathbb{I} / n$ ) semi-circle law.

Having noticed that $\rho-\mathbb{I} / n$ and $\frac{1}{n \sqrt{s}} G$ have the same limiting spectral distribution, we need to deduce that this implies their asymptotic equivalence in the sense of (8) . This is done in two steps. First, we 
point out that known results about convergence to the semicircle law can be subsumed in the language of the so-called $\infty$-Wasserstein distance (in random matrix theory, such results are usually stated in a rather weak form). Next we show that this (combined with unitary invariance) implies that the expectations of the gauges $\|\cdot\|_{K}$ must be asymptotically the same for both ensembles; this part of the argument is based on Appendix $\mathrm{A}$ and on the so-called majorization theory. We emphasize that the latter step is delicate since there are no uniform assumptions on continuity of the gauge $\|\cdot\|_{K}$.

Remark 3.2. While the formulation of Proposition 3.1 focuses on the regime when $n$ and $s / n$ tend to infinity, the proof can be adapted to other situations. For example, one can show that, for any $\alpha>0$,

$$
0<\inf _{s \geqslant \alpha n} c_{n, s} \leqslant \sup _{s \geqslant \alpha n} C_{n, s}<+\infty .
$$

This allows to establish a threshold phenomenon even for properties - in place of separability - for which $s_{0}(d) \simeq d^{2}$. However, in that case the argument does not yield the sharp threshold property, i.e., involving arbitrary $\varepsilon>0$. See Section 7.4 for more comments on related issues.

We now return to the proof of assertions (i) and (ii) of Theorem 2.3 , Applying Proposition 3.1 for $K=\mathcal{S}_{0}$ and using the definition (7) of $s_{0}(d)$, we obtain that (when $d$ and $s / d^{2}$ tend to infinity)

$$
\mathbf{E}\left\|\rho-\frac{\mathbb{I}}{n}\right\|_{\mathcal{S}_{0}} \sim \sqrt{\frac{s_{0}(d)}{s}} .
$$

Since a state $\rho$ is separable when $\|\rho-\mathbb{I} / n\|_{\mathcal{S}_{0}} \leqslant 1$ and entangled when $\|\rho-\mathbb{I} / n\|_{\mathcal{S}_{0}}>1$, this suggests that separability is typical when $s>s_{0}(d)$ and entanglement is typical when $s<s_{0}(d)$. This will be made rigorous through the next proposition; its proof is based on concentration of measure (reviewed in Section 2.2.).

Proposition 3.3. Let $s \geqslant n$, let $K \subset \mathcal{D}\left(\mathbf{C}^{n}\right)$ be a convex body with inradius $r$, and let $\rho$ be a random state with distribution $\mu_{n, s}$. Let $M$ be the median of $\|\rho-\mathbb{I} / n\|_{K_{0}}$, with $K_{0}=K-\mathbb{I} / n$. Then, for every $\eta>0$,

$$
\mathbf{P}\left(\left|\left\|\rho-\frac{\mathbb{I}}{n}\right\|_{K_{0}}-M\right| \geqslant \eta\right) \lesssim \exp (-c s)+\exp \left(-c n^{2} s r^{2} \eta^{2}\right)
$$

Proof. Let $\rho$ be a random state on $\mathbf{C}^{n}$ with distribution $\mu_{n, s}$. By definition, $\rho$ has the same distribution as

$$
\operatorname{tr}_{\mathbf{C}^{s}}|\psi\rangle\langle\psi|,
$$

where $\psi$ is uniformly distributed on the unit sphere in $\mathbf{C}^{n} \otimes \mathbf{C}^{s}$. Equivalently, $\rho$ has the same distribution as $A A^{\dagger}$, where $A$ is an $n \times s$ matrix uniformly distributed on the Hilbert-Schmidt sphere $S_{H S}$ (this is not immediately obvious, but can be verified by a straightforward calculation; also, $S_{H S}$ can be identified with the real sphere $S^{2 n s-1}$ ). Consider the function $f: S_{H S} \rightarrow \mathbf{R}$ defined by

$$
f(A)=\left\|A A^{\dagger}-\frac{\mathbb{I}}{n}\right\|_{K}
$$

Lemma 3.4. For every $t>0$, denote by $\Omega_{t}$ the subset

$$
\Omega_{t}=\left\{A \in S_{H S}:\|A\|_{\infty} \leqslant t\right\} .
$$

Then the Lipschitz constant of the restriction of $f$ to $\Omega_{t}$ is bounded by $2 t / r$.

Remark 3.5. In particular, taking $t=1$, one obtains that the global Lipschitz constant of $f$ is bounded by $2 / r$. This implies that any two central values for $f$ differ by at most $C /(r \sqrt{n s})$. 
Proof. The function $f$ is the composition of several operations:

- the map $A \mapsto\|A\|_{K}$, which is $1 / r$-Lipschitz with respect to the Hilbert-Schmidt norm.

- the map $A \mapsto A-\mathbb{I} / n$, which is an isometry for the Hilbert-Schmidt norm,

- the map $A \mapsto A A^{\dagger}$, whose Lipschitz constant can be estimated by the following chain of inequalities

$$
\begin{aligned}
\left\|A A^{\dagger}-B B^{\dagger}\right\|_{2} & \leqslant\left\|A\left(A^{\dagger}-B^{\dagger}\right)+(A-B) B^{\dagger}\right\|_{2} \\
& \leqslant\|A\|_{\infty}\left\|A^{\dagger}-B^{\dagger}\right\|_{2}+\|A-B\|_{2}\left\|B^{\dagger}\right\|_{\infty} \\
& \leqslant\left(\|A\|_{\infty}+\|B\|_{\infty}\right)\|A-B\|_{2} .
\end{aligned}
$$

In particular, if $A, B \in \Omega$, we obtain $\left\|A A^{\dagger}-B B^{\dagger}\right\|_{2} \leqslant 2 t\|A-B\|_{2}$.

We now apply Lemma 2.2, and we obtain that for every $\eta>0$,

$$
\mathbf{P}(|f-M| \geqslant \eta) \lesssim \mathbf{P}\left(S_{H S} \backslash \Omega_{t}\right)+\exp \left(-c_{1} n s \eta^{2}(2 t / r)^{-2}\right) .
$$

If we choose $t=3 / \sqrt{n}$, then $\mathbf{P}\left(S_{H S} \backslash \Omega_{t}\right) \lesssim \exp (-c s)$ (this follows from an elementary net argument, as explained in Lemma 6 and Appendix B of [5]) and the result follows.

We shall now show how to use Propositions 3.1 and 3.3 to conclude the proof of assertions (i) and (ii) of Theorem 2.3. For any integers $d$ and $s$, we define $\pi_{d, s}$ as the probability that a random state on $\mathbf{C}^{d} \otimes \mathbf{C}^{d}$ with distribution $\mu_{d^{2}, s}$ is separable. We first show that $\pi_{d, s}$ is decreasing with respect to $d$.

Lemma 3.6. Let $s, d_{1}, d_{2}$ be integers such that $d_{1} \leqslant d_{2}$. Then

$$
\pi_{d_{2}, s} \leqslant \pi_{d_{1}, s} .
$$

Proof. Identify $\mathbf{C}^{d_{1}}$ as a subspace of $\mathbf{C}^{d_{2}}$, and let $Q: \mathbf{C}^{d_{2}} \rightarrow \mathbf{C}^{d_{1}}$ be the orthogonal projection. Then, $\mathbf{C}^{d_{1}} \otimes \mathbf{C}^{d_{1}} \subset \mathbf{C}^{d_{2}} \otimes \mathbf{C}^{d_{2}}$ is the range of the projection $P=Q \otimes Q$. Let $\rho_{2}$ be a random state on $\mathbf{C}^{d_{2}} \otimes \mathbf{C}^{d_{2}}$ with distribution $\mu_{d_{2}^{2}, s}^{2}$. Then the state

$$
\rho_{1}:=\frac{P \rho_{2} P}{\operatorname{tr} P \rho_{2} P}
$$

is a random state on $\mathbf{C}^{d_{1}} \otimes \mathbf{C}^{d_{1}}$ with distribution $\mu_{d_{1}^{2}, s}$ (this is obvious if we realize $\rho_{2}$ as $G G^{\dagger} / \operatorname{tr} G G^{\dagger}$, where $G$ is a $d_{2}^{2} \times s$ random matrix with i.i.d. $N_{\mathbf{C}}(0,1)$ entries). The inequality follows from the fact that, given the relation (12), the separability of $\rho_{2}$ implies the separability of $\rho_{1}$ (local operations cannot create entanglement).

Remark 3.7. Another natural problem is whether $s_{1} \leqslant s_{2}$ implies $\pi_{d, s_{1}} \leqslant \pi_{d, s_{2}}$, i.e. whether the probability that a random induced state is separable always increases with the dimension of the environment. We do not know the answer to this question (see Section 7.5).

Proof of assertions (i) and (ii) of Theorem 2.3. We first address part (i): fix $\varepsilon>0$, then for any $s$ and $d$ satisfying the condition $s \leqslant(1-\varepsilon) s_{0}(d)$, we have to show that

$$
\pi_{d, s} \leqslant 2 \exp \left(-c(\varepsilon) d^{3}\right) .
$$

We start by establishing a slightly different estimate

$$
\pi_{d, s} \leqslant 2 \exp (-c(\varepsilon) s)
$$

which is stronger than (13) in the crucial range $s \gtrsim d^{3}$. The case $s=o\left(d^{3}\right)$ of (13) can be then deduced formally using Lemma 3.6 and other known facts. We relegate the details to Section 7.5 since 
the qualitative information provided by (13) and (14) is essentially the same; the only reason why we did not state the Theorem with the bound (14) is that it would give a misleading impression that the genericity of entanglement wanes as $s$ decreases, while in fact the opposite is true.

In view of Lemma 3.6, to prove (14) it is enough to consider the case when, for a given $s, d=d_{s}$ is the minimal integer satisfying $s \leqslant(1-\varepsilon) s_{0}(d)$. In particular, we have then

$$
\lim _{s \rightarrow \infty} s / d^{2}=+\infty
$$

and we are in the asymptotic regime described in Proposition 3.1, Let $M_{s}$ (resp. $E_{s}$ ) be the median (resp. the expectation) of $\left\|\rho-\mathbb{I} / d^{2}\right\|_{\mathcal{S}_{0}}$, when $\rho$ is a random state on $\mathbf{C}^{d} \otimes \mathbf{C}^{d}$ with distribution $\rho_{d^{2}, s}$. Applying Proposition 3.1, we have, when $s \rightarrow \infty$,

$$
E_{s} \sim \sqrt{\frac{s_{0}(d)}{s}} \sim \frac{1}{\sqrt{1-\varepsilon}}
$$

We now apply Proposition 3.3 to the convex body $K=\mathcal{S}_{0}$ (its inradius is of order $1 / n$, cf. Table 11). This gives, for any $\eta>0$,

$$
\mathbf{P}\left(||\left|\rho-\mathbb{I} / d^{2} \|_{\mathcal{S}_{0}}-M_{s}\right| \geqslant \eta\right) \lesssim \exp (-c s)+\exp \left(-c s \eta^{2}\right) .
$$

Moreover, we have $\left|M_{s}-E_{s}\right| \lesssim d / \sqrt{s}$ (cf. the remark following Lemma 3.4). Since $d / \sqrt{s}$ tends to 0 , $M_{s}$ is also equivalent to $1 / \sqrt{1-\varepsilon}$. We now choose $\eta>0$ such that $1 / \sqrt{1-\varepsilon}-\eta>1$, and we obtain that for $s$ large enough

$$
\pi_{d, s}=\mathbf{P}\left(\left\|\rho-\mathbb{I} / d^{2}\right\|_{\mathcal{S}_{0}} \leqslant 1\right) \leqslant C \exp (-c s)+C \exp \left(-c s \eta^{2}\right)
$$

Hence there are constants $C^{\prime}, c^{\prime}(\varepsilon)>0 \mathrm{such}$, for every $s$, we have the inequality $\pi_{d, s} \leqslant C^{\prime} \exp \left(-c^{\prime}(\varepsilon) s\right)$ (small values of $s$ are taken into account by adjusting the constants if necessary). A priori we may have $C^{\prime}>2$, but in that case the bound (14) follows with $c(\varepsilon)=c^{\prime}(\varepsilon) / \log _{2} C^{\prime}$. This shows part (i) of Theorem 2.3. except for the fine points related to the difference between the tail estimates (13) and (14), which will be clarified in Section 7.5. The part (ii) is proved in the same way as (14).

\section{Estimation of $s_{0}$}

This section is devoted to the proof of the inequalities

$$
d^{3} \lesssim s_{0}(d) \lesssim d^{3} \log ^{2} d
$$

which comprise the first assertion of Theorem 2.3. By formula (7) defining $s_{0}$, these inequalities are equivalent to

$$
d^{7 / 2} \lesssim \mathbf{E}\|G\|_{\mathcal{S}_{0}} \lesssim d^{7 / 2} \log d
$$

That is, we need to estimate the Gaussian average value of the gauge $\|\cdot\|_{\mathcal{S}_{0}}$.

It turns out that evaluating (or even estimating) $\mathbf{E}\|G\|_{\mathcal{S}_{0}}$ directly is not easy. This may conceivably be related to the fact that computing $\|\cdot\|_{\mathcal{S}_{0}}$ is an NP-hard problem [17]. Alternatively, we may note that $\mathbf{E}\|G\|_{\mathcal{S}_{0}}$ is directly related (via (3)) to the mean width of $\mathcal{S}_{0}^{\circ}$. Since there is a canonical link between duality of cones and duality of bases of cones (see [48], Lemma 1), it follows that any question about $\mathcal{S}_{0}^{\circ}$ is equivalent to a question about the cone of block-positive matrices and - via the Choi-Jamiołkowski isomorphism - to a question about the notoriously difficult to study cone of positivity-preserving maps on $\mathcal{M}_{d}$, the algebra of $d \times d$ complex matrices (see [9], or sections II and III in [48] for details).

The approach which succeeds is to proceed through a duality argument. First, we estimate $\mathbf{E}\|G\|_{\mathcal{S}_{0}^{\circ}}$ (or, equivalently, the mean width of $\mathcal{S}_{0}$ ), which is an easier task. Then, we use a general theorem saying 
that for any "well-balanced" symmetric convex body one can deduce the average of the norm from the average of the dual norm, with a multiplicative error logarithmic in the dimension. Since these aspects of the theory of high-dimensional convex geometry require the hypothesis of symmetry, we intoduce the following symmetrization of the convex body $\mathcal{S}_{0}$

$$
\mathcal{S}_{\text {sym }}=-\mathcal{S}_{0} \cap \mathcal{S}_{0}
$$

We first check that the relevant geometric parameters are essentially unchanged by this symmetrization procedure.

Proposition 4.1. The convex bodies $\mathcal{S}_{0}$ and $\mathcal{S}_{\text {sym }}$ have

(i) comparable average gauge:

$$
\mathbf{E}\|G\|_{\mathcal{S}_{0}} \leqslant \mathbf{E}\|G\|_{\mathcal{S}_{\mathrm{sym}}} \leqslant 2 \mathbf{E}\|G\|_{\mathcal{S}_{0}},
$$

(ii) comparable volume radius:

$$
\frac{1}{2} \operatorname{vrad}\left(\mathcal{S}_{0}\right) \leqslant \operatorname{vrad}\left(\mathcal{S}_{\text {sym }}\right) \leqslant \operatorname{vrad}\left(\mathcal{S}_{0}\right),
$$

(iii) comparable mean width:

$$
w\left(\mathcal{S}_{0}\right) \simeq w\left(\mathcal{S}_{\mathrm{sym}}\right) \simeq n^{-3 / 4},
$$

(iv) the same inradius, equal to $(n(n-1))^{-1 / 2}$. However, the outradius of $\mathcal{S}_{\text {sym }}$ is bounded by $1 / \sqrt{n}$, while the outradius of $\mathcal{S}_{0}$ is of order 1.

Proof. We have

$$
\|A\|_{\mathcal{S}_{\mathrm{sym}}}=\max \left(\|A\|_{\mathcal{S}_{0}},\|-A\|_{\mathcal{S}_{0}}\right) \leqslant\|A\|_{\mathcal{S}_{0}}+\|-A\|_{\mathcal{S}_{0}},
$$

from which (i) follows, because the distribution of $G$ is symmetric.

The fact that volume is preserved is less elementary. Several results in this direction are due to RogersShephard (cf. [39]); they additionally assert that the worst case occurs when the body is a simplex. For the present symmetrization, we use the following inequality which is a variation on the Rogers-Shephard inequality

Proposition 4.2. If $K \subset \mathbf{R}^{m}$ is a convex body with center of mass at the origin, then

$$
\operatorname{vol}(-K \cap K) \geqslant 2^{-m} \operatorname{vol}(K) .
$$

A proof can be found in [33] (Corollary 3 ). The factor $2^{-m}$ is not likely to be sharp; it is again tempting to conjecture that the simplex is the extremal case, but this seems to be unknown.

We apply Proposition 4.2 to $\mathcal{S}_{0}$ (to check that 0 is the center of mass of $\mathcal{S}_{0}$, average over local unitaries) and obtain

$$
\operatorname{vrad}\left(\mathcal{S}_{\text {sym }}\right) \geqslant \frac{1}{2} \operatorname{vrad}\left(\mathcal{S}_{0}\right)
$$

which shows (ii) (the other inequality is trivial).

For (iii), we already know (cf. Table 1) that

$$
\operatorname{vrad}\left(\mathcal{S}_{0}\right) \simeq w\left(\mathcal{S}_{0}\right) \simeq n^{-3 / 4} .
$$

We therefore have the following chain of inequalities (the first is trivial, the third is (ii) and the last is Urysohn's inequality (2))

$$
w\left(\mathcal{S}_{\text {sym }}\right) \leqslant w\left(\mathcal{S}_{0}\right) \simeq \operatorname{vrad}\left(\mathcal{S}_{0}\right) \simeq \operatorname{vrad}\left(\mathcal{S}_{\text {sym }}\right) \leqslant w\left(\mathcal{S}_{\text {sym }}\right) .
$$

Therefore all these quantities are comparable, and (iii) follows. 
For (iv), the statement about inradius is trivial. On the other hand, any matrix $A \in \mathcal{S}_{0}$ satisfies $A \geqslant-\mathbb{I} / n$. This implies that any $A \in \mathcal{S}_{\text {sym }}$ satisfies $-\mathbb{I} / n \leqslant A \leqslant \mathbb{I} / n$, or $\|A\|_{\infty} \leqslant 1 / n$, and therefore the outradius of $\mathcal{S}_{\text {sym }}$ is bounded by $1 / \sqrt{n}$. This completes the proof of Proposition 4.1 ,

The required estimates for $s_{0}$ follow now from the next lemma

Lemma 4.3. In the notation of the present section, we have

$$
n^{2} \lesssim \mathbf{E}\|G\|_{\mathcal{S}_{\text {sym }}} \cdot \mathbf{E}\|G\|_{\mathcal{S}_{\text {sym }}^{\circ}} \lesssim n^{2} \log n
$$

Indeed, in view of (3) and (4), Proposition 4.1(iii) implies that

$$
\mathbf{E}\|G\|_{\mathcal{S}_{\mathrm{sym}}^{\circ}} \simeq n^{1 / 4} \text {. }
$$

From Lemma 4.3, we infer that

$$
n^{7 / 4} \lesssim \mathbf{E}\|G\|_{\mathcal{S}_{\text {sym }}} \lesssim n^{7 / 4} \log n
$$

and the inequalities (16) follow from part (i) of Proposition 4.1 (recall that $n=d^{2}$, hence $\log n=2 \log d$ ). Since (16) was equivalent to the first assertion of Theorem 2.3, to conclude the proof of the Theorem it remains to show Lemma 4.3 .

Proof of Lemma 4.3. The proof makes use of the $\ell$-position of convex bodies, which is reviewed in Appendix D here we just mention that a body is in the $\ell$-position if it is isotropic is some precise technical sense. (Note, however, that there are different notions of isotropy and the $\ell$-position is not the most common one.) We also point out that the first inequality in Lemma 4.3 is elementary, see the last paragraph in Appendix D.

The upper inequality will follow from the $M M^{*}$-estimate (Proposition D.4), which is valid for any symmetric convex body that is in the $\ell$-position. If we knew that the convex body $\mathcal{S}_{\text {sym }}$ was in the $\ell$-position, the result would be just an instance of the $M M^{*}$-estimate (applied with $m=n^{2}-1$, which implies $\log m<2 \log n$ ). However, there are not enough symmetries present to conclude automatically that $\mathcal{S}_{\text {sym }}$ is in the $\ell$-position.

We proceed as follows. Let $E \subset \mathcal{M}_{n}^{s a, 0}$ be the subspace spanned by the operators of the form $\sigma_{1} \otimes \sigma_{2}$, where $\sigma_{1}$ and $\sigma_{2}$ are self-adjoint operators with trace 0 on $\mathbf{C}^{d}$. Let $F$ be the orthogonal complement of $E$ in $\mathcal{M}_{n}^{s a, 0}$. We then have

$$
F=\left\{\sigma_{1} \otimes \mathbb{I}: \operatorname{tr} \sigma_{1}=0\right\} \oplus\left\{\mathbb{I} \otimes \sigma_{2}: \operatorname{tr} \sigma_{2}=0\right\}=: F_{1} \oplus F_{2} .
$$

Clearly $\operatorname{dim} E=(n-1)^{2}$ and $\operatorname{dim} F=2 n-2$.

Let $u: \mathcal{M}_{n}^{s a, 0} \rightarrow \mathcal{M}_{n}^{s a, 0}$ be a linear map such that $u\left(\mathcal{S}_{\text {sym }}\right)$ is in the $\ell$-position. By combining Lemma C.1 with Lemma D.3 from the Appendices, we may assume that $u$ has the form

$$
u=P_{E}+\left(\mathbf{0}_{E} \oplus v\right)
$$

for some (positive definite operator) $v: F \rightarrow F$, where $\mathbf{0}_{E}$ is the zero operator on $E$. The ideal property of the $\ell$-norm implies that

$$
\ell_{\mathcal{S}_{\mathrm{sym}}}\left(P_{E}\right)=\ell_{\mathcal{S}_{\mathrm{sym}}}\left(u P_{E}\right) \leqslant \ell_{\mathcal{S}_{\mathrm{sym}}}(u)
$$

and similarly for $\ell_{\mathcal{S}_{\mathrm{sym}}^{\circ}}\left(P_{E}\right)$. By the $M M^{*}$-estimate (Proposition D.4), we know that

$$
\ell_{\mathcal{S}_{\mathrm{sym}}}(u) \ell_{\mathcal{S}_{\mathrm{sym}}^{\circ}}\left(u^{-1}\right) \lesssim n^{2} \log n,
$$

and therefore $\ell_{\mathcal{S}_{\mathrm{sym}}}\left(P_{E}\right) \ell_{\mathcal{S}_{\mathrm{sym}}^{\circ}}\left(P_{E}\right) \lesssim n^{2} \log n$ (note that $u^{-1}=P_{E}+\left(\mathbf{0}_{E} \oplus v^{-1}\right)$ ). To deduce similar estimates for $\mathbb{I}$ in place of $P_{E}$ we need the following observation. 
Claim 4.4. $\ell_{\mathcal{S}_{\mathrm{sym}}}\left(P_{F}\right)=o\left(\ell_{\mathcal{S}_{\mathrm{sym}}}(\mathbb{I})\right)$ and $\ell_{\mathcal{S}_{\mathrm{sym}}^{\circ}}\left(P_{F}\right)=o\left(\ell_{\mathcal{S}_{\mathrm{sym}}^{\circ}}(\mathbb{I})\right)$.

Once the claim is proved, using the triangle inequality to bound $\ell_{\mathcal{S}_{\text {sym }}}(\mathbb{I}) \leqslant \ell_{\mathcal{S}_{\text {sym }}}\left(P_{E}\right)+\ell_{\mathcal{S}_{\text {sym }}}\left(P_{F}\right)$ (and similarly for $\left.\ell_{\mathcal{S}_{\mathrm{sym}}^{\circ}}\right)$, we obtain $\ell_{\mathcal{S}_{\mathrm{sym}}}(\mathbb{I}) \ell_{\mathcal{S}_{\mathrm{sym}}^{\circ}}(\mathbb{I}) \lesssim n^{2} \log n$, which is equivalent to the second inequality in the assertion of Lemma 4.3 .

Proof of Claim 4.4. We use the estimates on the inradius and the outradius of $\mathcal{S}_{\text {sym }}$ (see Proposition 4.1(iv)) to deduce the following inequalities (recall that $\gamma_{m}$ was defined in (41))

$$
\begin{gathered}
\ell_{\mathcal{S}_{\mathrm{sym}}}\left(P_{F}\right)=\mathbf{E}\left\|P_{F} G\right\|_{\mathcal{S}_{\mathrm{sym}}}=w_{G}\left(\left(\mathcal{S}_{\mathrm{sym}} \cap F\right)^{\circ}\right) \leqslant n \gamma_{\operatorname{dim} F} \lesssim n^{3 / 2}, \\
\ell_{\mathcal{S}_{\mathrm{sym}}^{\circ}}\left(P_{F}\right)=\mathbf{E}\left\|P_{F} G\right\|_{\mathcal{S}_{\mathrm{sym}}^{\circ}}=w_{G}\left(P_{F} \mathcal{S}_{\mathrm{sym}}\right) \leqslant n^{-1 / 2} \gamma_{\operatorname{dim} F} \lesssim 1 .
\end{gathered}
$$

On the other hand, we have $\ell_{\mathcal{S}_{\text {sym }}^{\circ}}(\mathbb{I}) \simeq n^{1 / 4}$ (by equation 17 ) and $\ell_{\mathcal{S}_{\text {sym }}}(\mathbb{I}) \gtrsim n^{7 / 4}$ (by the already shown lower estimate from Lemma 4.3). This proves Claim 4.4 and concludes the proof of Lemma 4.3, and hence of the first assertion of Theorem 2.3. Combined with the arguments in the preceding section, this concludes the proof of the Theorem.

Remark 4.5. In the proof of Claim 4.4, we upper-bounded the mean widths of the convex bodies $P_{F} K$ and $(K \cap F)^{\circ}$ by their outradii. This is sufficient for the present argument, but these estimates are far from optimal. A more refined analysis is performed in Lemma 5.4; it will be needed to handle the case of multipartite systems and unbalanced bipartite systems.

\section{Extension to the multipartite CASE}

In this section we consider the case of a multipartite system, on the Hilbert space $\mathcal{H}=\left(\mathbf{C}^{d}\right)^{\otimes k}$, and estimate the threshold for separability of random states in the asymptotic regime when $k \geqslant 2$ is fixed and $d$ tends to $+\infty$. We denote $n=d^{k}=\operatorname{dim} \mathcal{H}$ and $m=n^{2}-1=\operatorname{dim} \mathcal{D}(\mathcal{H})$. In this section, constants are allowed to depend on $k$; this is emphasized by writing $\lesssim_{k}, \simeq_{k}, o_{k}(\cdot)$ instead of $\lesssim, \simeq, o(\cdot)$.

The set of separable states on $\mathcal{H}$ is the subset of $\mathcal{D}(\mathcal{H})$ defined as

$$
\mathcal{S}(\mathcal{H})=\operatorname{conv}\left\{\left|\psi_{1} \otimes \cdots \otimes \psi_{k}\right\rangle\left\langle\psi_{1} \otimes \cdots \otimes \psi_{k}\left|: \psi_{i} \in \mathbf{C}^{d},\right| \psi_{i}\right|=1, i=1, \cdots k\right\} .
$$

As in the bipartite case, our argument requires estimates on geometric parameters associated to $\mathcal{S}(\mathcal{H})$, given in the next table. The statement about the inradius was proved in [19], and the statements about the mean width and the volume radius were obtained in [4].

TABLE 2. Radii of $\mathcal{S}$ for $\left(\mathbf{C}^{d}\right)^{\otimes k}$, where $n=d^{k}$.

\begin{tabular}{|c|c|c|c|c|}
\hline & inradius & volume radius & mean width & outradius \\
\hline $\mathcal{S}\left(\left(\mathbf{C}^{d}\right)^{\otimes k}\right)$ & $\simeq_{k} n^{-1}$ & $\simeq_{k} n^{-1+\frac{1}{2 k}}$ & $\simeq_{k} n^{-1+\frac{1}{2 k}}$ & $=\sqrt{(n-1) / n}$ \\
\hline
\end{tabular}

The following is a multipartite version of Theorem 2.3 .

Theorem 5.1. For every integer $k \geqslant 2$, there are effectively computable positive constants $c_{k}$ and $C_{k}$, depending only on $k$, and a function $s_{0}(k, d)$ satisfying

$$
c_{k} d^{2 k-1} \leqslant s_{0}(k, d) \leqslant C_{k} d^{2 k-1} \log ^{2} d,
$$

such that if $\rho$ is a random state on $\left(\mathbf{C}^{d}\right)^{\otimes k}$ distributed according to the measure $\mu_{d^{k}, s}$, then for every $\varepsilon>0$, 
(i) If $s \leqslant(1-\varepsilon) s_{0}(k, d)$, then

$$
\mathbf{P}(\rho \text { is separable }) \leqslant 2 \exp (-c(\varepsilon) s) .
$$

(ii) If $s \geqslant(1+\varepsilon) s_{0}(k, d)$, then

$$
\mathbf{P}(\rho \text { is entangled }) \leqslant 2 \exp (-c(\varepsilon) s) .
$$

Here $c(\varepsilon)$ is a positive constant depending on $\varepsilon$ and on $k$.

The proof of Theorem 5.1 is completely parallel to the bipartite case, except for one point where a slightly finer analysis is required. We set $\mathcal{S}_{0}=\mathcal{S}_{0}\left(\left(\mathbf{C}^{d}\right)^{\otimes k}\right)=\mathcal{S}\left(\left(\mathbf{C}^{d}\right)^{\otimes k}\right)-\mathbb{I} / n, \mathcal{S}_{\text {sym }}=-\mathcal{S}_{0} \cap \mathcal{S}_{0}$ and define the threshold $s_{0}(k, d)$ as

$$
s_{0}(k, d):=\left(\frac{\mathbf{E}\|G\|_{\mathcal{S}_{0}}}{d^{k}}\right)^{2} \sim w\left(\mathcal{S}_{0}^{\circ}\right)^{2} .
$$

We first need to show that the estimates (18) hold. We only sketch the proof. First, Proposition 4.1 carries over verbatim to the multipartite setting, and implies (using the formula from Table 2) that

$$
\mathbf{E}\|G\|_{\mathcal{S}_{\text {sym }}^{\circ}} \simeq_{k} n^{\frac{1}{2 k}} .
$$

The multipartite version of Lemma 4.3 is

Lemma 5.2. In the notation of the present section, we have

$$
n^{2} \lesssim \mathbf{E}\|G\|_{\mathcal{S}_{\mathrm{sym}}} \cdot \mathbf{E}\|G\|_{\mathcal{S}_{\mathrm{sym}}^{\circ}} \lesssim_{k} n^{2} \log n .
$$

Proof. We proceed as follows. Let $E \subset \mathcal{M}_{n}^{s a, 0}$ be the subspace spanned by the products of trace zero operators, and $F$ be the orthogonal complement of $E$. We have $\operatorname{dim} E=\left(d^{2}-1\right)^{k}$ and $\operatorname{dim} F=d^{2 k}-1-$ $\left(d^{2}-1\right)^{k} \simeq_{k} n^{2-2 / k}$. Let $u: \mathcal{M}_{n}^{s a, 0} \rightarrow \mathcal{M}_{n}^{s a, 0}$ be a linear map such that $u\left(\mathcal{S}_{\text {sym }}\right)$ is in the $\ell$-position. By the results in Appendices $\mathrm{D}$ and $\mathrm{C}$ we may assume that $u$ has the form

$$
u=P_{E}+\left(\mathbf{0}_{E} \oplus v\right)
$$

for some positive map $v: F \rightarrow F$. As in the bipartite case, the $M M^{*}$-estimate and the ideal property of the $\ell$-norm imply that $\ell_{\mathcal{S}_{\text {sym }}}\left(P_{E}\right) \ell_{\mathcal{S}_{\text {sym }}^{\circ}}\left(P_{E}\right) \lesssim n^{2} \log n$, and so Lemma 5.2 will follow from the following

Claim 5.3. $\ell_{\mathcal{S}_{\mathrm{sym}}}\left(P_{F}\right)=o_{k}\left(\ell_{\mathcal{S}_{\mathrm{sym}}}(\mathbb{I})\right)=o_{k}\left(n^{2-\frac{1}{2 k}}\right)$ and $\ell_{\mathcal{S}_{\mathrm{sym}}^{\circ}}\left(P_{F}\right)=o_{k}\left(\ell_{\mathcal{S}_{\mathrm{sym}}^{\circ}}(\mathbb{I})\right)=o_{k}\left(n^{\frac{1}{2 k}}\right)$.

To show the Claim, we note first that the inradius of $\mathcal{S}_{\text {sym }}$ equals the inradius of $\mathcal{S}_{0}$ and is of order $n^{-1}$ (from Table 22). Hence $\|\cdot\|_{\mathcal{S}_{\mathrm{sym}}} \lesssim_{k} n|\cdot|$ and so

$$
\ell_{\mathcal{S}_{\text {sym }}}\left(P_{F}\right)=\mathbf{E}\left\|P_{F} G\right\|_{\mathcal{S}_{\text {sym }}} \lesssim_{k} n \mathbf{E}\left|P_{F} G\right|=n \gamma_{\operatorname{dim} F} \simeq_{k} n^{2-1 / k}
$$

This implies the first part of the Claim. The second part requires a little finer analysis. For $1 \leqslant j \leqslant k$, denote by $F_{j}$ the following subspace of $\mathcal{M}_{n}^{s a, 0}$

$$
F_{j}=\left(\mathcal{M}_{s a}\left(\mathbf{C}^{d}\right) \otimes \cdots \otimes \mathcal{M}_{s a}\left(\mathbf{C}^{d}\right) \otimes \mathbf{R I} \otimes \mathcal{M}_{s a}\left(\mathbf{C}^{d}\right) \otimes \cdots \otimes \mathcal{M}_{s a}\left(\mathbf{C}^{d}\right)\right) \cap \mathcal{M}_{n}^{s a, 0}
$$

where the factor $\mathbf{R I}$ appears at position $j$. Since $F \subset \bigoplus_{j} F_{j}$, it suffices to prove that $\ell_{\mathcal{S}_{\text {sym }}^{\circ}}\left(P_{F_{j}}\right)=$ $o_{k}\left(n^{\frac{1}{2 k}}\right)$-it follows from the ideal property that $\ell_{\mathcal{S}_{\text {sym }}^{\circ}}\left(P_{V}\right) \leqslant \ell_{\mathcal{S}_{\text {sym }}^{\circ}}\left(P_{V^{\prime}}\right)$ whenever $V \subset V^{\prime}$. By symmetry we may assume $j=1$. We have

$$
\ell_{\mathcal{S}_{\mathrm{sym}}^{\circ}}\left(P_{F_{1}}\right)=\mathbf{E}\left\|P_{F_{1}} G\right\|_{\mathcal{S}_{\mathrm{sym}}^{\circ}}=w_{G}\left(P_{F_{1}} \mathcal{S}_{\mathrm{sym}}\right) \leqslant w_{G}\left(P_{F_{1}} \mathcal{S}_{0}\right) .
$$


It turns out that the convex body $P_{F_{1}} \mathcal{S}_{0}$ has a simple description.

Lemma 5.4. For every $k \geqslant 2$, we have

$$
P_{F_{1}} \mathcal{S}_{0}\left(\left(\mathbf{C}^{d}\right)^{\otimes k}\right)=\mathcal{S}_{0}\left(\left(\mathbf{C}^{d}\right)^{\otimes k}\right) \cap F_{1}=\mathbb{I} / d \otimes \mathcal{S}_{0}\left(\left(\mathbf{C}^{d}\right)^{\otimes(k-1)}\right),
$$

with the notation $x \otimes K=\{x \otimes y: y \in K\}$.

Proof. The inclusions $\supset$ are immediate. Conversely, let $\rho \in \mathcal{S}\left(\left(\mathbf{C}^{d}\right)^{\otimes k}\right)$. Starting from a separable decomposition

we obtain

$$
\rho=\sum \lambda_{i} \rho_{i}^{(1)} \otimes \cdots \otimes \rho_{i}^{(k)}
$$

$$
P_{F_{1}} \rho=\sum \lambda_{i} \mathbb{I} / d \otimes \rho_{i}^{(2)} \otimes \cdots \otimes \rho_{i}^{(k)}-\mathbb{I} / d^{k}=\mathbb{I} / d \otimes\left(\sigma-\mathbb{I} / d^{k-1}\right),
$$

with $\sigma \in \mathcal{S}\left(\left(\mathbf{C}^{d}\right)^{\otimes(k-1)}\right)$ defined as

$$
\sigma=\sum \lambda_{i} \rho_{i}^{(2)} \otimes \cdots \otimes \rho_{i}^{(k)} .
$$

It follows that $P_{F_{1}} \mathcal{S}_{0}\left(\left(\mathbf{C}^{d}\right)^{\otimes k}\right)=P_{F_{1}} \mathcal{S}\left(\left(\mathbf{C}^{d}\right)^{\otimes k}\right) \subset \mathbb{I} / d \otimes \mathcal{S}_{0}\left(\left(\mathbf{C}^{d}\right)^{\otimes(k-1)}\right)$.

Remark 5.5. In the case $k=2$, the convex body $\mathcal{S}_{0}\left(\left(\mathbf{C}^{d}\right)^{\otimes(k-1)}\right)$ should be interpreted as $\mathcal{D}_{0}\left(\mathbf{C}^{d}\right)$.

We now return to the proof of Claim 5.2, From Lemma 5.4, it is very easy to compute $w_{G}\left(P_{F_{1}} \mathcal{S}_{0}\right)$. Since $\|\mathbb{I} / d\|_{2}=1 / \sqrt{d}$, the convex bodies $P_{F_{1}} \mathcal{S}_{0}\left(\left(\mathbf{C}^{d}\right)^{\otimes k}\right)$ and $\frac{1}{\sqrt{d}} \mathcal{S}_{0}\left(\left(\mathbf{C}^{d}\right)^{\otimes(k-1)}\right)$ are congruent, hence have the same Gaussian mean width. Since $w_{G}\left(\mathcal{S}_{0}\left(\left(\mathbf{C}^{d}\right)^{\otimes(k-1)}\right)\right) \simeq_{k} \sqrt{d}$ (see Table 2), we obtain from (19) that

$$
\ell_{\mathcal{S}_{\mathrm{sym}}^{\circ}}\left(P_{F_{1}}\right) \leqslant w_{G}\left(P_{F_{1}} \mathcal{S}_{0}\left(\left(\mathbf{C}^{d}\right)^{\otimes k}\right)\right)=\frac{1}{\sqrt{d}} w_{G}\left(\mathcal{S}_{0}\left(\left(\mathbf{C}^{d}\right)^{\otimes(k-1)}\right)\right) \simeq_{k} 1
$$

which completes the proof of the Claim, and hence that of Lemma 5.2

The rest of the proof of Theorem 5.1 follows by mimicking the argument in the bipartite case given in Sections 3 and 4 .

\section{Generic entanglement: an alternative approach}

In this section we sketch an alternative proof that entanglement on $\mathbf{C}^{d} \otimes \mathbf{C}^{d}$ is generic when $s$ is sufficiently smaller than $d^{3}$ (Theorem 2.3(i)). The proof also yields a similar statement addressing two different regimes of the the multipartite case, including the one dealt with in Theorem 5.1(i). In its present form, the argument does not show that separability is generic for larger $s$. However, we present it here since it leads to sharp estimates in the "small ball" regime (i.e., when the probability of separability is very small), and since it is much more straightforward.

The proof is based on analyzing directly the density (6) of $\mu_{n, s}$ and specifically on the following

Lemma 6.1. Let $s \geqslant n$ with $\log s \ll n$. Then there exists a universal constant $C>0$, such that for any measurable subset $\mathcal{K} \subset \mathcal{D}=\mathcal{D}\left(\mathbf{C}^{n}\right)$,

$$
\mu_{n, s}(\mathcal{K})^{\frac{1}{m}} \leqslant C \sqrt{\frac{s}{n}} \mu_{n, n}(\mathcal{K})^{\frac{1}{m}}
$$

where $m=n^{2}-1=\operatorname{dim} \mathcal{D}$. 
Assuming the lemma and remembering that $\mu_{n, n}$ is just the Lebesgue measure (the Hilbert-Schmidt volume), it is easy to deduce (an estimate stronger than) the assertion (i) of Theorem 2.3 from known results. Indeed, if $n=d^{2}$ and $\mathcal{K}=\mathcal{S}=\mathcal{S}\left(\mathbf{C}^{d} \otimes \mathbf{C}^{d}\right)$, we can read from Table 1 that

$$
\mu_{n, n}(\mathcal{S})^{\frac{1}{m}}=\left(\frac{\operatorname{vol} \mathcal{S}}{\operatorname{vol} \mathcal{D}}\right)^{\frac{1}{m}}=\frac{\operatorname{vrad}(\mathcal{S})}{\operatorname{vrad}(\mathcal{D})} \simeq \frac{n^{-3 / 4}}{n^{-1 / 2}}=n^{-1 / 4}
$$

Consequently,

$$
\mu_{n, s}(\mathcal{S}) \leqslant\left(C_{1} \sqrt{\frac{s}{n}} n^{-1 / 4}\right)^{m}=\left(C_{1}^{2} \frac{s}{d^{3}}\right)^{m / 2},
$$

and so the probability of separability is very small if $s<C_{1}^{-2} n^{3 / 2}=C_{1}^{-2} d^{3}$. (Note that the exponent is here of order $d^{4}$, as opposed to $d^{3}$ in Theorem 2.3(i).)

It is also straightforward to obtain in the same way estimates on the threshold value of $s$ for multipartite entanglement (i.e., for all $k \geqslant 2$ ). Sharp bounds for $\frac{\mathrm{vol} \mathcal{S}}{\mathrm{vol} \mathcal{D}}$ in the multipartite case were derived in [46, 4]:

Proposition 6.2. Let $\mathcal{H}=\left(\mathbf{C}^{d}\right)^{\otimes k}$ and let $\mathcal{S}(\mathcal{H})$ be the corresponding set of $k$-partite separable states. Then

(i) $\left(\frac{\operatorname{vol} \mathcal{S}(\mathcal{H})}{\operatorname{vol} \mathcal{D}(\mathcal{H})}\right)^{\frac{1}{m}} \lesssim(k \log k)^{1 / 2} n^{-\frac{1}{2}+\frac{1}{2 k}} ;$

(ii) $\left(\frac{\operatorname{vol} \mathcal{S}(\mathcal{H})}{\operatorname{vol} \mathcal{D}(\mathcal{H})}\right)^{\frac{1}{m}} \lesssim\left(\frac{d k \log k}{n^{1+\beta_{d}}}\right)^{1 / 2}$, where $\beta_{d}=\log _{d}\left(1+\frac{1}{d}\right)-\frac{1}{d^{2}} \log _{d}(d+1)$.

The two bounds in the Proposition reflect emphasis on two regimes, fixed $k$ and large $d$ (small number of large subsystems) and fixed $d$ and large $k$ (large number of small subsystems). When combined with Lemma 6.1, they lead immediately to the following

Theorem 6.3. In the notation of Proposition 6.2, let $s \geqslant n=d^{k}$ and let $\rho$ be a random state on $\mathcal{H}$ distributed according to the measure $\mu_{n, s}$. Then $\mathbf{P}(\rho \in \mathcal{S}(\mathcal{H}))$ is (exponentially in $m=n^{2}-1$ ) small if $s \leqslant c(k) n^{2-1 / k}$ or $s \leqslant c(k) c^{\prime}(d) n^{2+\beta_{d}}$, where $c(k) \gtrsim(k \log k)^{-1}$ and $c^{\prime}(d) \gtrsim d^{-1}$.

As the above bounds on the threshold values of $s$ may appear not-very-transparent, we will make them explicit in some special cases. First, in the bipartite case $k=2$ (two large subsystems), we again recover the bound of order $n^{3 / 2}=d^{3}$. In the tripartite case $k=3$, entanglement is generic if $s \leqslant c n^{5 / 3}$. If $d=2$ and $k$ is large ( $k$ qubits), the bound on the threshold value of $s$ is (modulo factors of smaller order) $n^{2+\beta_{2}}$, where $\beta_{2} \approx 0.18872$ (we also have the equality $n^{2+\beta_{2}}=2^{k} 3^{3 k / 4}$, but the latter expression is again not-so-transparent). The numerical constants implicit in the $\gtrsim, \lesssim$ notation in the Proposition and in the Theorem are effectively computable and can be recovered from the discussion following Theorems 1 and 2 in [4] and from the proof of Lemma 6.1.

Proof of Lemma 6.1 (sketch). The argument follows the lines of [54], where related questions were considered. Since, by the arithmetic-geometric mean inequality, $\operatorname{det} \rho \leqslant n^{-n}$ for $\rho \in \mathcal{D}\left(\mathbf{C}^{n}\right)$, formula (6) implies

$$
\mu_{n, s}(\mathcal{K})=\frac{1}{Z_{n, s}} \int_{\mathcal{K}}(\operatorname{det} \rho)^{s-n} d \rho \leqslant \frac{1}{Z_{n, s}} \int_{\mathcal{K}} n^{-n(s-n)} d \rho=n^{-n(s-n)} \frac{Z_{n, n}}{Z_{n, s}} \mu_{n, n}(\mathcal{K})
$$


Hence the Lemma reduces to showing that $\left(n^{-n(s-n)} \frac{Z_{n, n}}{Z_{n, s}}\right)^{\frac{1}{m}} \lesssim \sqrt{\frac{s}{n}}$ or, equivalently (we may replace $m=n^{2}-1$ in the exponent by $n^{2}$ as $\operatorname{long}$ as $\left.\log s \ll n\right)$,

$$
\left(\frac{Z_{n, n}}{Z_{n, s}}\right)^{\frac{1}{n^{2}}} \lesssim \frac{s^{1 / 2} n^{s / n}}{n^{3 / 2}}
$$

Explicit formulae for $Z_{n, s}$ are known (see [32, 56, 55, 9])

$$
Z_{n, s}=\frac{\sqrt{n}(2 \pi)^{n(n-1) / 2}}{\Gamma(s n)} \prod_{k=s-n+1}^{s} \Gamma(k) .
$$

We point out that these quantities are sometimes referred to in the literature as "the $\alpha$-volume" (with $\alpha=s-n+1)$. Also, the normalization factors are often calculated for densities on the Weyl chamber $\left\{\left(\lambda_{1}, \cdots, \lambda_{n}\right) \in \mathbf{R}^{n}: \sum_{i=1}^{n} \lambda_{i}=1, \lambda_{1} \geqslant \cdots \geqslant \lambda_{n} \geqslant 0\right\}$ in the simplex of eigenvalues rather than for densities on the set of states; the two quantities differ by the factor $(2 \pi)^{n(n-1) / 2} / \prod_{j=1}^{n} \Gamma(j)$, equal to the measure of the corresponding flag manifold.

What remains is a tedious but routine calculation based on the Stirling formula, used here in the form $\ln \Gamma(x)=x \ln x-x+O(\ln x)$. We have

$$
\begin{aligned}
\frac{\ln Z_{n, s}}{n^{2}}-\ln \sqrt{2 \pi} & \sim \frac{1}{n^{2}}\left(\sum_{k=s-n+1}^{s} \ln \Gamma(k)-\ln \Gamma(s n)\right) \\
& \sim \frac{1}{n^{2}}\left(\sum_{k=s-n+1}^{s}(k \ln k-k)-s n \ln (s n)+s n\right) \\
& \sim \frac{1}{n^{2}} \int_{k=s-n}^{s}(x \ln x-x) d x-\frac{s}{n} \ln (s n)+\frac{s}{n} \\
& =\frac{\frac{s^{2}}{2} \ln s-\frac{3 s^{2}}{4}-\left(\frac{(s-n)^{2}}{2} \ln (s-n)-\frac{3(s-n)^{2}}{4}\right)}{n^{2}}-\frac{s}{n} \ln (s n)+\frac{s}{n} .
\end{aligned}
$$

We now use the bound $\ln (s-n)=\ln s+\ln \left(1-\frac{n}{s}\right) \leqslant \ln s-\frac{n}{s}$ to obtain, after simplifications,

$$
\frac{\ln Z_{n, s}}{n^{2}} \gtrsim \ln \sqrt{2 \pi}-\frac{1}{4}-\frac{s}{n} \ln n-\frac{1}{2} \ln s
$$

and hence, after exponentiating,

$$
\left(Z_{n, s}\right)^{\frac{1}{n^{2}}} \gtrsim n^{-s / n} s^{-1 / 2}
$$

Similarly, but in a much simpler way, we are led to

$$
\left(Z_{n, n}\right)^{\frac{1}{n^{2}}} \sim \sqrt{2 \pi} e^{1 / 4} n^{-3 / 2} .
$$

(This calculation was already performed in [46], where the equivalent formula $\operatorname{vrad} \mathcal{D}\left(\mathbf{C}^{n}\right) \sim e^{-1 / 4} n^{-1 / 2}$ was derived.) Combining the last two estimates we obtain (20). 


\section{Miscellaneous Remarks and loose EndS}

7.1. Threshold from zero to nonzero probability of separability. In this paper we estimated the threshold for separability in terms of the ancilla dimension, and showed that the probability of entanglement changes dramatically from nearly one to nearly zero around this threshold. A seemingly related question (but actually very different) is to ask for which ancilla dimensions the probability of separability is exactly zero. Here is a summary of our knowledge about this problem. (We do not claim originality.)

Proposition 7.1. Let $\rho$ be a random state on $\mathbf{C}^{d} \otimes \mathbf{C}^{d}$ distributed according to the probability measure $\mu_{d^{2}, s}$.

(i) If $s \geqslant d^{2}$, then $0<\mathbf{P}(\rho$ is separable $)<1$.

(ii) If $s \leqslant(d-1)^{2}$, then $\mathbf{P}(\rho$ is separable $)=0$.

(iii) If $d=2$, then $0<\mathbf{P}(\rho$ is separable $)<1$ for $s \geqslant 3$ and $\mathbf{P}(\rho$ is separable $)=0$ for $s \leqslant 2$.

Proof. (i) follows from both $\mathcal{S}$ and $\mathcal{D} \backslash \mathcal{S}$ having nonempty interior and from the density of $\mu_{n, s}$ (6) being strictly positive in the interior of $\mathcal{D}$ for $s \geqslant n$. (ii) is a simple combination of Corollary 3.5 in [50], which asserts that a random subspace of dimension $s$ in $\mathbf{C}^{d} \otimes \mathbf{C}^{d}$ almost surely contains no product vector when $s \leqslant(d-1)^{2}$, and of [23], which points out that a separable state must have a product vector in its range. Next, the only instances of (iii) which are not covered by the two preceding parts are $s=2,3$; these are slightly more delicate. If $s=3$, then the relevant measure $\mu_{4,3}$ (note that $n=d^{2}=4$ here) is concentrated on the boundary of $\mathcal{D}$. However, since the eigenvalues of $M M^{\dagger}$ (for a $4 \times 3$ matrix $M$ ) are the same as those of $M^{\dagger} M$ plus an additional 0 , and since the distributions of $M M^{\dagger}$ and $M^{\dagger} M$ are unitarily invariant, it follows that $M M^{\dagger}$ has a density with respect to the surface measure which is (modulo a normalization factor) $\operatorname{det}\left(M^{\dagger} M\right)$, and in particular is nonzero on a set of full (surface) measure. On the other hand, it follows from [47] that the probability of separability on the boundary of $\mathcal{D}$ (with respect to the surface measure) equals $\frac{1}{2} \frac{\mathrm{vol} \mathcal{S}}{\mathrm{vol} \mathcal{D}} \in\left(0, \frac{1}{2}\right)$, which combined with the preceding remark yields the conclusion. Similarly, if $d=2$ and $s=2$, the relevant measure $\mu_{4,2}$ is supported on the set of states of rank (at most) 2. The question of generic separability of such states was studied in [41] (see also [2]). While the measure considered in [41] is apparently different from $\mu_{4,2}$, they are both induced by parametrizations of the set of states of rank 2 which are smooth outside of a subset of lower dimension, and all such measures are mutually absolutely continuous. Accordingly, our conclusion follows from Corollary 4 in [41. (The authors thank Mary Beth Ruskai for bringing the paper [41] to their attention.)

7.2. The unbalanced case: $\mathcal{H}=\mathbf{C}^{d_{1}} \otimes \mathbf{C}^{d_{2}}, d_{1} \neq d_{2}$. A result analogous to Theorem 2.3 holds, with the threshold $s_{0}\left(d_{1}, d_{2}\right)$ verifying

$$
\text { cn } \min \left\{d_{1}, d_{2}\right\} \leqslant s_{0}\left(d_{1}, d_{2}\right) \leqslant C n(\log n)^{2} \min \left\{d_{1}, d_{2}\right\},
$$

where $n=d_{1} d_{2}$. To show this, let us try to retrace the arguments from the balanced case. We may assume $d_{1} \leqslant d_{2}$. First, one checks that the argument from [4] yields $w(\mathcal{S})=w\left(\mathcal{S}_{0}\right) \simeq d_{2}^{1 / 2} / n=\left(n d_{1}\right)^{-1 / 2}$ (note that if we are only interested in the bipartite case, the $\lesssim$ part follows rather easily from Lemma 2 in [4]). The needed estimates for $w\left(\mathcal{S}_{0}^{\circ}\right)$ follow then word by word, except that the upper estimate requires a slightly more careful analysis which we detail now. If, as in the balanced case, we denote $\mathcal{S}_{\text {sym }}=-\mathcal{S}_{0} \cap \mathcal{S}_{0}$, we have

$$
w_{G}\left(\mathcal{S}_{\mathrm{sym}}\right) \simeq \sqrt{d_{2}}
$$


Let $E, F_{1}$ and $F_{2}$ be the subspaces appearing in the proof of Lemma 4.3. The conclusion will follow if we prove that (for $i=1,2$ )

$$
\ell_{\mathcal{S}_{\mathrm{sym}}}\left(P_{F_{i}}\right) \leqslant \frac{1}{3} \ell_{\mathcal{S}_{\mathrm{sym}}}(\mathbb{I})=\frac{1}{3} w_{G}\left(\mathcal{S}_{\mathrm{sym}}^{\circ}\right) \quad ; \quad \ell_{\mathcal{S}_{\mathrm{sym}}^{\circ}}\left(P_{F_{i}}\right) \leqslant \frac{1}{3} \ell_{\mathcal{S}_{\mathrm{sym}}^{\circ}}(\mathbb{I})=\frac{1}{3} w_{G}\left(\mathcal{S}_{\mathrm{sym}}\right) .
$$

Indeed, since $\ell_{\mathcal{S}_{\text {sym }}}(\mathbb{I}) \leqslant \ell_{\mathcal{S}_{\text {sym }}}\left(P_{E}\right)+\ell_{\mathcal{S}_{\text {sym }}}\left(P_{F_{1}}\right)+\ell_{\mathcal{S}_{\text {sym }}}\left(P_{F_{2}}\right)$, it follows from (22) that $\ell_{\mathcal{S}_{\text {sym }}}(\mathbb{I}) \leqslant 3 \ell_{\mathcal{S}_{\text {sym }}}\left(P_{E}\right)$, and similarly for $\ell_{\mathcal{S}_{\mathrm{sym}}^{\circ}}$, and therefore

$$
w_{G}\left(\mathcal{S}_{\mathrm{sym}}\right) w_{G}\left(\mathcal{S}_{\mathrm{sym}}^{\circ}\right)=\ell_{\mathcal{S}_{\mathrm{sym}}}(\mathbb{I}) \ell_{\mathcal{S}_{\mathrm{sym}}^{\circ}}(\mathbb{I}) \leqslant 9 \ell_{\mathcal{S}_{\mathrm{sym}}}\left(P_{E}\right) \ell_{\mathcal{S}_{\mathrm{sym}}^{\circ}}\left(P_{E}\right) \lesssim n^{2} \log n
$$

by the $M M^{*}$-estimate.

Using Proposition D.1 and the unbalanced version of Lemma 5.4, we obtain

$$
\ell_{\mathcal{S}_{\mathrm{sym}}}\left(P_{F_{1}}\right)=w_{G}\left(\left(\mathcal{S}_{\mathrm{sym}} \cap F_{1}\right)^{\circ}\right)=w_{G}\left(\left[d_{1}^{-1 / 2} \mathcal{D}_{\mathrm{sym}}\left(\mathbf{C}^{d_{2}}\right)\right]^{\circ}\right) \simeq \sqrt{d_{1}} d_{2}^{3 / 2}
$$

where $\mathcal{D}_{\text {sym }}=-\mathcal{D}_{0} \cap \mathcal{D}_{0}$. Similarly,

$$
\ell_{\mathcal{S}_{\text {sym }}^{\circ}}\left(P_{F_{1}}\right)=w_{G}\left(P_{F_{1}} \mathcal{S}_{\text {sym }}\right) \leqslant w_{G}\left(d_{1}^{-1 / 2} \mathcal{D}\left(\mathbf{C}^{d_{2}}\right)\right) \simeq \sqrt{d_{2} / d_{1}}
$$

Note that the equivalences $w_{G}\left(\mathcal{D}_{\text {sym }}^{\circ}\right) \simeq w_{G}\left(\mathcal{D}_{0}^{\circ}\right)$ and $w_{G}\left(\mathcal{D}_{\text {sym }}\right) \simeq w_{G}(\mathcal{D})$, and the values of these parameters, were determined in Proposition 4.1, (i) and (iii). Analogous estimates hold for $F_{2}$ with the role of $d_{1}$ and $d_{2}$ exchanged. We conclude that the conditions (22) are satisfied unless $d_{1}$ is smaller than some absolute constant.

On the other hand, if $2 \leqslant \min \left\{d_{1}, d_{2}\right\} \leqslant C$, then $\mathcal{D}_{0} \subset\left(C^{2}-1\right) \mathcal{S}_{0}$ (cf. [18], Corollary 5; by looking at the Schmidt decomposition of a pure state it is easy to see that any bound valid with $d_{1}=d_{2}=C-$ the case considered in [18] - holds also when $\min \left\{d_{1}, d_{2}\right\} \leqslant C$ ). Accordingly, the widths of $\mathcal{S}, \mathcal{S}_{0}^{\circ}$ are the same as those of $\mathcal{D}, \mathcal{D}_{0}^{\circ}$ "up to universal multiplicative constants" and the calculation becomes trivial (in this case, there is no log factor in the upper bound for $s_{0}\left(d_{1}, d_{2}\right)$ ).

7.3. Calculating the precise order of the mean width of $\mathcal{S}^{\circ}$, or is the $\log$ necessary. It is conceivable that the presence of logarithmic factors in Lemma 4.3, and hence in Theorems 2.3 and 5.1 , is due to the fact that we appeal to a result about general convex bodies (Proposition D.4) rather than calculate $w\left(\mathcal{S}_{0}^{\circ}\right)$ directly (or to the lack of precision in the upper bound from Proposition D.4). Due to the fundamental nature of the separability/entanglement dichotomy, the question about the precise order of $w\left(\mathcal{S}_{0}^{\circ}\right)$ is interesting by itself. It may be worthwhile to note that for the convex body $L=\mathcal{D}_{0}\left(\mathbf{C}^{n}\right)$, the product $w(L) w\left(L^{\circ}\right)$ is majorized by a universal constant, even though the argument via $\ell$-position necessarily leads to an upper bound which is $\gtrsim \sqrt{\log n}$.

7.4. Thresholds for other "standard" properties of quantum states. Our method can be generalized to estimate thresholds for other properties of random induced states (beyond separability), provided the set of states with this property is a convex subset $K \subset \mathcal{D}$ and has some minimal invariance properties (such as being fixed under conjugation with local unitaries). In any such application one needs to estimate the mean width of $K^{\circ}$, or at least the volume of $K$ in order to adapt the argument from Section 6 .

One natural example of $K$ is the set $\mathcal{P} \mathcal{P} \mathcal{T}\left(\mathbf{C}^{d} \otimes \mathbf{C}^{d}\right)$ of states with positive partial transpose (the volume radius and the mean width of which were estimated in [4] and shown to be much larger than those of $\mathcal{S}$ ). Since $\mathcal{P} \mathcal{P} \mathcal{T}=\mathcal{D} \cap T(\mathcal{D})$, where $T$ is the partial transpose, it follows easily (cf. Proposition 4.1(i)) that $w\left(\mathcal{P} \mathcal{P} \mathcal{T}_{0}^{\circ}\right) \leqslant 2 w\left(\mathcal{D}_{0}^{\circ}\right) \simeq d$, whence $($ cf. (7) $) s_{0} \simeq d^{2}$. However, this is less precise than the result from [3] where it was shown - using completely different techniques - that a sharp threshold for PPT occurs at $s_{0} \sim 4 d^{2}$. (While a more careful argument using concentration of measure shows that $w\left(\mathcal{P} \mathcal{P} \mathcal{T}_{0}^{\circ}\right) \sim 2 d$, 
in our approach we will always lose an additional multiplicative constant when using Proposition 3.1 Similarly, while it is likely that our argument based on majorization may be modified to show existence of a sharp threshold also in the regime $s_{0} \simeq d^{2}$, it is not at all clear how to retrieve this way the exact multiplicative factor 4.)

A very interesting point is that there is a whole range of parameters, when the ancilla dimension $s$ satisfies (for an arbitrary $\varepsilon>0$ and an appropriate $c>0$ )

$$
(4+\varepsilon) d^{2} \leqslant s \leqslant c d^{3},
$$

where random induced states on $\mathbf{C}^{d} \otimes \mathbf{C}^{d}$ are - for large $d$ - generically bound entangled, i.e. entangled and PPT (hence non-distillable [25]).

7.5. Improving the probability estimates, and sharpness of the threshold.. Denote (as in Section (3) by $\pi_{d, s}$ the probability that a random state on $\mathbf{C}^{d} \otimes \mathbf{C}^{d}$ with distribution $\mu_{d^{2}, s}$ is separable. In Section 3 we showed that, if $s \leqslant(1-\varepsilon) s_{0}(d)$, then (14) holds, i.e.,

$$
\pi_{d, s} \leqslant 2 \exp (-c(\varepsilon) s) .
$$

The above probability estimate is not optimal, both in its dependence on $s$ and in the dependence on $\varepsilon$ that can be retrieved from the argument. The following conjecture sounds reasonable (the larger the environment is, the more uncommon entanglement is) and would formally imply the bound $\pi_{d, s} \leqslant$ $2 \exp \left(-c(\varepsilon) s_{0}(d)\right)$, which better agrees with physical heuristics and is formally stronger than both (14) and the assertion of Theorem 2.3 (i).

Conjecture 7.2. For any $d \geqslant 2$, the function $s \mapsto \pi_{d, s}$ is non-increasing.

While we do not prove this conjecture, the next simple lemma is a partial result in this direction.

Lemma 7.3. For every $d, s$, we have the inequality $\pi_{2 d, s} \leqslant \pi_{d, 4 s}$.

Proof. Identify $\mathbf{C}^{2 d}$ with $\mathbf{C}^{2} \otimes \mathbf{C}^{d}$, and let $\tau: \mathcal{D}\left(\mathbf{C}^{2 d} \otimes \mathbf{C}^{2 d}\right) \rightarrow \mathcal{D}\left(\mathbf{C}^{d} \otimes \mathbf{C}^{d}\right)$ be the partial trace over $\mathbf{C}^{2} \otimes \mathbf{C}^{2}$. Let $\rho$ be a random state on $\mathbf{C}^{2 d} \otimes \mathbf{C}^{2 d}$ with distribution $\mu_{4 d^{2}, s}$. Then $\tau(\rho)$ is a random state on $\mathbf{C}^{d} \otimes \mathbf{C}^{d}$ with distribution $\mu_{d^{2}, 4 s}$, and

$$
\rho \text { separable } \Longrightarrow \tau(\rho) \text { separable. }
$$

This shows the inequality $\pi_{2 d, s} \leqslant \pi_{d, 4 s}$.

There are other ways to improve the probability estimate in various ranges. First, note that $\pi_{d, s}=0$ if $s \leqslant(d-1)^{2}$ (see Section 7.1). Second, Theorem 6.3 implies that, for some absolute constants $c, c_{1}>0$, $\pi_{d, s} \lesssim \exp \left(-c d^{4}\right)$ whenever $d^{2} \leqslant s \leqslant c_{1} d^{3}$. This establishes in particular the bound $\pi_{d, s} \leqslant 2 \exp \left(-c(\varepsilon) d^{3}\right)$ asserted in Theorem 2.3 (i), except for the narrow range $(d-1)^{2}<s<d^{2}$. This exceptional range can be handled as follows. Set $d_{1}=\lfloor(d+1) / 2\rfloor$; then $\pi_{d, s} \leqslant \pi_{d_{1}, s}$ by Lemma 3.6. On the other hand, $s>(d-1)^{2} \geqslant d_{1}^{2}$ and (if $d$ is sufficiently large) $s<d^{2} \leqslant c_{1} d_{1}^{3}$, so Theorem 6.3 applies and shows that $\pi_{d_{1}, s} \lesssim \exp \left(-c d_{1}^{4}\right) \leqslant \exp \left(-c d^{4} / 16\right)$. Combining the estimates we conclude that the bound $\pi_{d, s} \lesssim \exp \left(-c d^{4}\right)$ extends to the entire range $s \leqslant c_{1} d^{3}$ (perhaps with a different constant $c$ ). When combined with the argument from Section 3, this completes the proof of Theorem 2.3 (i) as originally stated.

Estimates for probabilities in Theorem 2.3 and similar statements translate directly into assertions about sharpness of the entanglement-separability threshold at $s_{0}=s_{0}(d)$ : the increase in the ancilla dimension $s$ needed for the induced state to "flip" from generic entanglement to generic separability. As 
stated, Theorem 2.3 asserts that the increase is $o\left(s_{0}\right)$. Retracing the argument would allow to come up with an explicit (and clearly suboptimal) bound, apparently of the order of $s_{0}^{\theta}$ for some $\theta \in(0,1)$ (with $\theta$ rather close to 1). On the other hand, finding precise order is likely a difficult "small ball" problem (cf. [30]). So, instead of pursuing such calculations, we sketch a heuristic argument which suggests the limits of our approach, which may be not far from the actual behavior.

Our analysis shows that the sharpness of the threshold $s_{0}$ depends on a combination of two effects: (i) the decay of $\mathbf{E}\left\|\rho_{d^{2}, s}-\frac{\mathbb{I}}{n}\right\|_{\mathcal{S}_{0}}$ as a functions of $s$ and (ii) the concentration of $\left\|\rho_{d^{2}, s}-\frac{\mathbb{I}}{n}\right\|_{\mathcal{S}_{0}}$ around its mean (or median). We do know (from (9) ) that the former is approximately $\phi(s):=\sqrt{\frac{s_{0}}{s}}$, and from Proposition 3.3 (see (15) ) that $\left\|\rho_{d^{2}, s}-\frac{\mathbb{I}}{n}\right\|_{\mathcal{S}_{0}}$ is concentrated on an interval whose length is of order $\frac{1}{\sqrt{s}}$ : choosing as $\eta:=\frac{a}{\sqrt{s}}$, where $a$ is sufficiently large, makes the right hand side of (15) small. A simple calculation shows now that the increase in $s$ needed to reduce the value of $\phi(s)$ from $1+\eta$ to $1-\eta$ is of order $s^{1 / 2}$. The lack of rigor in this calculation stems from the approximation given by $\phi(s)$ not being known to be valid up to the precision of order $\eta$.

7.6. Regularity of the threshold function, and proof of Corollary 2.4. Theorem 2.3] is a statement about sharp transition from generic entanglement to generic separability as the dimension of the ancilla $s$ increases while the dimension $n=d^{2}$ of the system itself remains fixed. Clearly, this implies that a similar phenomenon takes place also with fixed $s$ and variable $d$. However, without any additional information about regularity of the threshold function $s_{0}(d)$, one can not formally infer that - for example - a sharp transition occurs also in this new setting.

While we do not have a complete picture of the regularity of $s_{0}(d)$, or of the associated probabilities $\pi_{d, s}$, we do have some information (Lemmas 3.6 and 7.3), which is enough to deduce Corollary 2.4.

As noted already in the Introduction, the setting of Corollary 2.4 (i.e., $N$ particles with $D$ levels each and two subsystems of $k$ particles each) is modeled by a random induced state on $\mathbf{C}^{d} \otimes \mathbf{C}^{d}$ with $d=D^{k}$ and $s=D^{N-2 k}$. Thus we need to show that the sequence $p_{k}:=\pi_{D^{k}, D^{N-2 k}}$ has the following property

(i) for some small $\delta>0$ (which quantifies the "near 0" and "near 1" probabilities) and for some $k_{0} \sim N / 5$ (the threshold value of $k$ ), $p_{k}>1-\delta$ if $k<k_{0}$ and $p_{k}<\delta$ if $k>k_{0}$.

Except for determining the value of $k_{0}$, this is equivalent to the following

(ii) if $p_{k} \leqslant 1-\delta$, then $p_{k+1}<\delta$.

We note that a slight generalization of Lemma 7.3, with 2 replaced by an arbitrary $D$ and 4 by $D^{2}$, implies that the sequence $\left(p_{k}\right)$ is non-increasing; this is not necessary for our argument, but nevertheless reassuring. Also, if $k$ is substantially smaller than $N / 5$, then $N-2 k$ is substantially larger than $3 k$ and so $s=D^{N-2 k}$ is substantially larger than $D^{3 k}=d^{3}$ and, consequently - by Theorem 2.3 - $p_{k}$ is close to 1. Similarly, if $k$ is substantially larger than $N / 5, p_{k}$ is close to 0 . Accordingly, there is no doubt that the transition from $p_{k} \approx 1$ to $p_{k} \approx 0$ does indeed occur as $k$ increases, and that it occurs when $k \sim N / 5$. The only point that needs to be made is the sharpness of the transition.

To that end, note that - by Theorem 2.3 - a property similar to (i) and (ii) holds for the sequence $q_{i}:=\pi_{d, D^{i}}$ for any fixed $d:$ if $D^{i} \leqslant(1-\varepsilon) s_{0}(d)$, then $\pi_{d, D^{i}}<\delta$ and if $D^{i} \geqslant(1+\varepsilon) s_{0}(d)$, then $\pi_{d, D^{i}}>1-\delta$ (as long as $\delta>2 \exp (-c(\varepsilon) s)$ for the appropriate values of $s$ ), and $(1-\varepsilon) s_{0}(d)<D^{i}<(1+\varepsilon) s_{0}(d)$ may happen at most for one value of $i \sim \log _{D}\left(s_{0}(d)\right) \sim 3 \log _{D} d$ (there will be no such $i$ at all, unless $\log _{D}\left(s_{0}(d)\right)$ is close to an integer).

To show that the condition (ii) above is satisfied for the sequence $\left(p_{k}\right)$, we argue as follows. If $p_{k}=$ $\pi_{D^{k}, D^{N-2 k}} \leqslant 1-\delta$, then - by Lemma 7.3 - also $\pi_{D^{k+1}, D^{N-2 k}} \leqslant 1-\delta$, and so the observation from the preceding paragraph applied with $q_{i}=\pi_{D^{k+1}, D^{i}}$ implies that $\pi_{D^{k+1}, D^{N-2 k-1}}<\delta$ and, applied one more 
time (this works if $\delta \leqslant 1-\delta$, or if $\delta \leqslant 1 / 2$, which may be readily assumed), that $p_{k+1}=\pi_{D^{k+1}, D^{N-2 k-2}}<\delta$, as needed.

\section{Appendix A. Majorization And $\infty$-Wasserstein distance}

We gather here some facts concerning the usual modes of convergence from probability, $\infty$-Wasserstein distance, and the concept of majorization. They will be used in the proof of Proposition 3.1 in the next appendix, but they are fairly general and independent of that particular application; we believe that stating them separately may be of reference value.

Definition A.1. Let $\mu_{1}, \mu_{2}$ be probability measures on $\mathbf{R}$. The $\infty$-Wasserstein distance is defined as

$$
d_{\infty}\left(\mu_{1}, \mu_{2}\right):=\inf \left\|X_{1}-X_{2}\right\|_{L^{\infty}},
$$

with infimum over all couples $\left(X_{1}, X_{2}\right)$ of random variables with (marginal) laws $\mu_{1}$ and $\mu_{2}$, defined on a common probability space. Similarly, if $Y_{1}, Y_{2}$ are real random variables, we will mean by $d_{\infty}\left(Y_{1}, Y_{2}\right)$ the $\infty$-Wasserstein distance between the laws of $Y_{1}$ and $Y_{2}$.

Note the following inequality: whenever $f: \mathbf{R} \rightarrow \mathbf{R}$ is a $L$-Lipschitz function and $X, Y$ are bounded random variables, we have

$$
|\mathbf{E} f(X)-\mathbf{E} f(Y)| \leqslant L d_{\infty}(X, Y) .
$$

The $\infty$-Wasserstein distance can be computed from cumulative distribution functions: if $F_{X}(t)=\mathbf{P}(X \leqslant$ $t$ ), then

$$
d_{\infty}(X, Y)=\inf \left\{\varepsilon>0: F_{X}(t-\varepsilon) \leqslant F_{Y}(t) \leqslant F_{X}(t+\varepsilon) \text { for all } t \in \mathbf{R}\right\} .
$$

The following lemma is elementary and can be proved by using the fact that the Lévy distance metrizes the weak convergence for probability measures (see e.g. [16], Section 4.3).

Lemma A.2. Let $Z$ be a random variable distributed according to a measure $\mu_{Z}$, with support equal to some bounded interval $[a, b]$. If $\left(Y_{n}\right)$ is a sequence of random variables, the following are equivalent:

(1) $d_{\infty}\left(Y_{n}, Z\right) \rightarrow 0$

(2) $Y_{n} \rightarrow Z$ weakly and $\sup Y_{n} \rightarrow b, \inf Y_{n} \rightarrow a$. 2

Note that the hypothesis on the support is crucial: the equivalence fails if the support is not connected.

Next, we will relate the $\infty$-Wasserstein distance to the concept of majorization, usually defined for vectors in $\mathbf{R}^{n}$. Given $x \in \mathbf{R}^{n}$ we will denote by $x^{\downarrow}$ the non-increasing rearrangement of $x$. If $x, y \in \mathbf{R}^{n, 0}$ (the hyperplane of sum 0 vectors in $\mathbf{R}^{n}$ ), we write $x \prec y$ if, for every $k \in\{1, \ldots, n\}$, we have

$$
\sum_{i=1}^{k} x_{i}^{\downarrow} \leqslant \sum_{i=1}^{k} y_{i}^{\downarrow}
$$

(note that for $k=n$ we always have equality). The following is well-known (see [10], Section II).

Proposition A.3. For $x, y \in \mathbf{R}^{n, 0}$ the following are equivalent.

(1) $x \prec y$,

(2) whenever $\phi$ is a permutationally invariant convex function on $\mathbf{R}^{n, 0}$, then $\phi(x) \leqslant \phi(y)$,

(3) For every $t \in \mathbf{R}$, we have $\sum_{i=1}^{n}\left|x_{i}-t\right| \leqslant \sum_{i=1}^{n}\left|y_{i}-t\right|$,

(4) $x$ can be written as a convex combination of coordinatewise permutations of $y$.

\footnotetext{
${ }^{2} \mathrm{By}$ inf and sup we really mean here essential inf and sup.
} 
We will need a quantitative version of the concept of majorization. If $x, x^{\prime} \in \mathbf{R}^{n, 0}$ with $x^{\prime} \neq 0$, we denote by $\delta\left(x, x^{\prime}\right)$ the smallest non-negative constant $t$ such that $x \prec t x^{\prime}$. In other words, $\delta\left(\cdot, x^{\prime}\right)$ is the gauge associated to the convex body obtained as the convex hull of coordinatewise permutations of $x^{\prime}$. The quantity $\delta\left(x, x^{\prime}\right)$ should not be thought of as a distance, but rather as the norm of a certain operator. We have the inequality $\delta\left(x, x^{\prime \prime}\right) \leqslant \delta\left(x, x^{\prime}\right) \delta\left(x^{\prime}, x^{\prime \prime}\right)$. More generally, if $\phi$ is any 1-homogeneous convex function on $\mathbf{R}^{n, 0}$ (for example, the gauge of a convex body) which is permutationally-invariant, then

$$
\phi(x) \leqslant \delta\left(x, x^{\prime}\right) \phi\left(x^{\prime}\right) .
$$

We can rephrase the concept of majorization in the language of " $n$-point empirical measures," i.e., the probability measures of the form $\nu_{x}=n^{-1} \sum_{k=1}^{n} \delta_{x_{k}}$. The restriction requiring that $x \in \mathbf{R}^{n, 0}$ translates into the underlying random variable having zero mean. Note that (by Proposition A.3.

$$
\delta(x, y)=\delta(y, x)=1 \Longleftrightarrow y \text { is a coordinatewise permutation of } x \Longleftrightarrow \nu_{x}=\nu_{y} .
$$

The following key observation connects majorization and convergence in $\infty$-Wasserstein distance, and may be of independent interest.

Proposition A.4. Let $Z$ be a non-constant bounded random variable with mean 0 and with distribution $\mu_{Z}$. Then, for every $\varepsilon>0$, there exists $\eta>0$ (depending on $Z$ and $\varepsilon$ ) such that for every $n$ and for all vectors $x, y \in \mathbf{R}^{n, 0}$ satisfying $d_{\infty}\left(\nu_{x}, \mu_{Z}\right) \leqslant \eta$ and $d_{\infty}\left(\nu_{y}, \mu_{Z}\right) \leqslant \eta$, we have $\delta(x, y) \leqslant 1+\varepsilon$.

Proof. Denote $a=\inf Z$ and $b=\sup Z$ (the hypotheses on $Z$ imply $a<0<b$ ). Let $\varepsilon>0$, and $x, y \in \mathbf{R}^{n, 0}$ such that $d_{\infty}\left(\nu_{x}, \mu_{Z}\right) \leqslant \eta$ and $d_{\infty}\left(\nu_{y}, \mu_{Z}\right) \leqslant \eta$. We must choose $\eta$ to ensure that $\delta(x, y) \leqslant 1+\varepsilon$ or, equivalently, $x \prec(1+\varepsilon) y$. By Proposition A.3, this is equivalent to

$$
\sum_{i=1}^{n}\left|x_{i}-t\right| \leqslant \sum_{i=1}^{n}\left|(1+\varepsilon) y_{i}-t\right| \quad \text { for all } t \in \mathbf{R}
$$

(where $x=\left(x_{i}\right)_{i=1}^{n}$ and $\left.y=\left(y_{i}\right)_{i=1}^{n}\right)$ or, in the language of $n$-point empirical measures,

$$
f_{0}(t):=\int|u-t| d \nu_{x}(u) \leqslant \int|(1+\varepsilon) u-t| d \nu_{y}(u)=: g_{0}(t)
$$

We first note that these conditions are automatically satisfied if $t \geqslant \max _{i} x_{i}$. Indeed, we have then $|u-t|=t-u$ for all $u$ in the support of $\nu_{x}$ and so the first integral above equals $\int t d \nu_{x}-\int u d \nu_{x}=t-0=t$. On the other hand, we always have $|(1+\varepsilon) u-t| \geqslant t-(1+\varepsilon) u$ and so the second integral is at least $\int t d \nu_{y}-\int(1+\varepsilon) u d \nu_{y}=t-(1+\varepsilon) 0=t$. Similar argument applies when $t \leqslant \min _{i} x_{i}$.

If we choose $\eta \leqslant \frac{\varepsilon}{2} \min (-a, b)$, then we have $(1+\varepsilon / 2) a \leqslant \min _{i} x_{i} \leqslant \max _{i} x_{i} \leqslant(1+\varepsilon / 2) b$ and therefore it suffices to show the inequality $f_{0} \leqslant g_{0}$ on the interval $((1+\varepsilon / 2) a,(1+\varepsilon / 2) b)$. To that end, we compare the functions $f_{0}$ and $g_{0}$ with the functions

$$
\begin{gathered}
f(t)=\mathbf{E}|Z-t|=\int|u-t| d \mu_{Z}(u), \\
g(t)=\mathbf{E}|(1+\varepsilon) Z-t|=\int|(1+\varepsilon) u-t| d \mu_{Z}(u) .
\end{gathered}
$$

Proposition A.4 will now follow from the following lemma.

Lemma A.5. In the above notation, $f(t)<g(t)$ for $t \in((1+\varepsilon) a,(1+\varepsilon) b)$. 
Indeed, since $f$ and $g$ are continuous, there is a number $\theta>0$ such that $f(t)<g(t)-\theta$ for every $t \in[(1+\varepsilon / 2) a,(1+\varepsilon / 2) b]$. On the other hand, by (23), we have $\left|f_{0}(t)-f(t)\right| \leqslant \eta$ and $\left|g_{0}(t)-g(t)\right| \leqslant(1+\varepsilon) \eta$ for any $t \in \mathbf{R}$. It remains to choose $\eta>0$ such that $(2+\varepsilon) \eta \leqslant \theta$ to guarantee that $f_{0} \leqslant g_{0}$ on $[(1+\varepsilon / 2) a,(1+\varepsilon / 2) b]$.

Proof of Lemma A.5. Assume first $t \geqslant 0$. We have

$$
g(t)-f(t)=\int(|(1+\varepsilon) u-t|-|u-t|) d \mu_{Z}(u)=\int(|(1+\varepsilon) u-t|-|u-t|+\varepsilon u) d \mu_{Z}(u) .
$$

It is now elementary to check that the last integrand is 0 if $u \leqslant t /(1+\varepsilon)$ and strictly positive if $u>t /(1+\varepsilon)$. Accordingly, the integral is always nonnegative, and it is strictly positive if the interval $(t /(1+\varepsilon), \infty)$ intersects the support of $\mu_{Z}$, that is exactly when $t /(1+\varepsilon)<b$, or $t<(1+\varepsilon) b$. The case $t \leqslant 0$ is handled similarly (or by a change of variable $u=-v$ ).

\section{Appendix B. GUE approximation to induced states: Proposition 3.1}

The strategy of the proof is as follows. First, we gather known facts from Random Matrix Theory which assert that, for the regime in question (i.e., $\left.n, \frac{n}{s} \rightarrow \infty\right)$ ), the appropriately normalized ensembles $\left(G_{n}\right)$ and $\left(\rho_{n, s}-\frac{\mathbb{I}}{n}\right)$ converge (in probability) to the same limit in the sense of non-commutative probability. Then we will show that - in the same regime - the expectations of the gauges $\|\cdot\|_{K}$ must be asymptotically the same for both ensembles, which is essentially the assertion of Proposition [3.1; this part of the argument will be based on the material from Appendix A.

We first set some notation. Recall that $\mathcal{M}_{n}^{s a}$ is the space of self-adjoint operators on $\mathbf{C}^{n}$ and $\mathcal{M}_{n}^{s a, 0}$ - the subspace of self-adjoint trace 0 operators. We denote by $\operatorname{sp}(A) \in \mathbf{R}^{n}$ the spectrum of an operator $A \in \mathcal{M}_{n}^{s a}$ (ordered in the increasing order for definiteness, but this is irrelevant). Note that $A \in \mathcal{M}_{n}^{s a, 0} \Longleftrightarrow \operatorname{sp}(A) \in$ $\mathbf{R}^{n, 0}$ (the hyperplane of sum zero vectors in $\mathbf{R}^{n}$ ). Conversely, if $x \in \mathbf{R}^{n}$, we denote by $\operatorname{Diag}(x) \in \mathcal{M}_{n}^{s a}$ the diagonal matrix built from $x$.

Recall that the standard semicircular distribution (or Wigner distribution) is the probability distribution $\mu_{s c}$ with density

$$
\frac{1}{2 \pi} \sqrt{4-x^{2}} \mathbf{1}_{|x| \leqslant 2}
$$

Note that a random variable $Z$ with semicircular distribution satisfies the hypotheses of Lemmas A.2 and Proposition A.4.

We are now in a position to state the needed facts from Random Matrix Theory. Recall that the $n$-point empirical measure associated to a vector $x=\left(x_{k}\right) \in \mathbf{R}^{n}$ is the measure $\nu_{x}=n^{-1} \sum_{k=1}^{n} \delta_{x_{k}}$.

Proposition B.1. For every $n$, let $G_{n}$ be an $n \times n \mathrm{GUE}^{0}$ random matrix, and let $\nu_{\mathrm{sp}\left(n^{-1 / 2} G_{n}\right)}$ be the rescaled empirical spectral distribution. When $n$ tends to infinity, the sequence $\left(\nu_{\mathrm{sp}\left(n^{-1 / 2} G_{n}\right)}\right)$ converges to $\mu_{s c}$, in probability, with respect to the $\infty$-Wasserstein distance.

Convergence in probability means that for any $\varepsilon>0, \lim _{n \rightarrow \infty} \mathbf{P}\left(d_{\infty}\left(\mu_{n}, \mu_{s c}\right)>\varepsilon\right)=0$.

Proposition B.2. For every $n, s$, let $A_{n, s}=\rho_{n, s}-\frac{\mathbb{I}}{n}$, where $\rho_{n, s}$ is a random state with distribution $\mu_{n, s}$, and let $\nu_{\mathrm{sp}\left(\sqrt{n s} A_{n, s)}\right.}$ be the rescaled empirical spectral distribution. When $n$ and $s / n$ tend to infinity, the

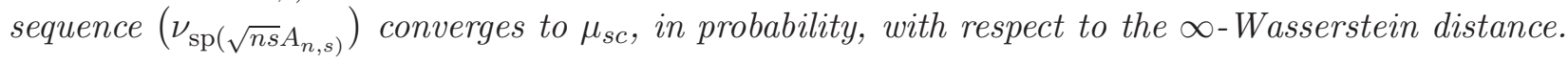


References for Propositions B.1 and B.2. By Lemma A.2, convergence to the semi-circle distribution with respect to the $\infty$-Wasserstein distance is equivalent to weak convergence and convergence of extreme eigenvalues. These statements appear separately in random matrix literature.

Wigner's famous result (the semi-circle law, see [1]) is that $\nu_{\mathrm{sp}\left(n^{-1 / 2} G_{n}\right)}$ converges weakly, in probability, towards $\mu_{s c}$. The standard setting is the case of GUE matrices, while here we consider $\mathrm{GUE}^{0}$ matrices (i.e., GUE matrices conditioned to have trace 0), but it is easily checked that this doesn't affect the limit distribution.

The statement about convergence of limit eigenvalues is also well-known. For example, it is known that $\mathbf{E}\left\|G_{n}\right\|_{\infty} \leqslant 2 \sqrt{n}$ (a proof for GUE matrices can be found in Appendix H of [46], and this extends to $\mathrm{GUE}^{0}$ via Jensen's inequality). Concentration of measure (e.g., the Gaussian version of Lemma 2.1) implies then that

$$
\mathbf{P}\left(\left\|G_{n} / \sqrt{n}\right\|_{\infty} \geqslant 2+t\right) \leqslant \exp \left(-n t^{2} / 2\right) .
$$

Some readers may be surprised by Proposition B.2 since $\rho_{n, s}$ is a (rescaled) Wishart ensemble, which is known to have the Marchenko-Pastur law as the limiting spectral distribution [31. However, that limit is obtained when $n, s \rightarrow \infty$ with the ratio $s / n \rightarrow \beta$ for some $\beta>0$, while here we have $s / n \rightarrow \infty$. For a non-rigorous but convincing and simple calculation, the reader may verify that when $\beta \rightarrow \infty$, the (appropriately rescaled) Marchenko-Pastur density does indeed converge to a (non-centered) semi-circular density.

For a rigorous argument, we refer to [7, where the weak convergence towards the semicircular distribution is proved (one should point out that this statement appeared implicitly already in [31]). The statement about limit eigenvalues, as well as tail inequalities analogous to (26), can be deduced from the techniques from [20] (see also [12], Theorem 2.7).

We point out that while the (rather difficult) memoir [20] is the ultimate reference on the subject of rectangular complex Gaussian matrices, and while it is indispensable for recovering very sharp results, the estimates we need here can be obtained by much simpler methods. The primary reason we are invoking [20] is that, historically, the topic of rectangular random matrices was studied only in the real case because of its relevance to statistics. However, most - but not all (cf. [13, §2.3) - arguments carry over to the complex case; in particular, the simple and elegant proof from [43] would be worthwhile to analyze in this regard. Finally, let us note that once the behavior of the limit eigenvalues is determined, the tail inequalities of the type (26) follow from the Gaussian concentration (see the comments following Lemma 2.1).

For every integer $n$, denote by $X_{n}^{\text {sc }} \in \mathbf{R}^{n, 0}$ the "ideally semicircular" vector, i.e. the vector $X_{n}^{\text {sc }}=$ $\left(X_{n, 1}^{\mathrm{sc}}, \ldots, X_{n, n}^{\mathrm{sc}}\right)$ such that

$$
F_{\mathrm{sc}}\left(X_{n, k}^{\mathrm{sc}}\right)=\frac{2 k-1}{2 n}
$$

where $F_{\mathrm{sc}}$ is the cumulative distribution function of standard semicircular distribution (note that the sum of coordinates is indeed zero). Obviously (see the beginning of Appendix $[\mathrm{A})$, the sequence $\left(\nu_{X_{n}^{\mathrm{sc}}}\right)$ converges to $\mu_{s c}$ in the $\infty$-Wasserstein distance.

Let $K$ be a convex body in $\mathcal{M}_{n}^{s a, 0}$ (containing 0 in the interior), with $\|\cdot\|_{K}$ the corresponding gauge function. Define a gauge $\phi_{K}$ on $\mathbf{R}^{n, 0}$ by setting

$$
\phi_{K}(x)=\int_{\mathcal{U}(n)}\left\|U \operatorname{Diag}(x) U^{\dagger}\right\|_{K} d U
$$


where the integral is taken with respect to the (normalized) Haar measure on the unitary group. Let $G_{n}$ be an $n \times n \mathrm{GUE}^{0}$ random matrix, and let $U \in \mathcal{U}(n)$ be a Haar-distributed random unitary matrix independent from $G_{n}$. By unitary invariance, $G_{n}$ has the same distribution as $U^{\dagger} \operatorname{Diag}\left(\operatorname{sp}\left(G_{n}\right)\right) U$. Therefore, we have

$$
\mathbf{E} \phi_{K}\left(\operatorname{sp}\left(G_{n}\right)\right)=\mathbf{E}\left\|G_{n}\right\|_{K}
$$

Since $\phi_{K}$ is convex and invariant under permutation of coordinates, it follows from (24) that $\phi_{K}(x) \leqslant$ $\delta(x, y) \phi_{K}(y)$ for every $x, y \in \mathbf{R}^{n, 0}$. In particular, if we introduce the random variables $\alpha_{n}=\delta\left(\operatorname{sp}\left(\frac{1}{\sqrt{n}} G_{n}\right), X_{n}^{\mathrm{sc}}\right)$ and $\beta_{n}=\delta\left(X_{n}^{\mathrm{sc}}, \operatorname{sp}\left(\frac{1}{\sqrt{n}} G_{n}\right)\right)$, we have

$$
\sqrt{n} \phi_{K}\left(X_{n}^{\mathrm{sc}}\right) \beta_{n}^{-1} \leqslant \phi_{K}\left(\operatorname{sp}\left(G_{n}\right)\right) \leqslant \sqrt{n} \phi_{K}\left(X_{n}^{\mathrm{sc}}\right) \alpha_{n} .
$$

Taking expectation, we obtain

$$
\sqrt{n} \phi_{K}\left(X_{n}^{\mathrm{sc}}\right) \mathbf{E} \beta_{n}^{-1} \leqslant \mathbf{E}\left\|G_{n}\right\|_{K} \leqslant \sqrt{n} \phi_{K}\left(X_{n}^{\mathrm{sc}}\right) \mathbf{E} \alpha_{n}
$$

Recall that $A_{n, s}=\rho_{n, s}-\frac{\mathbb{I}}{n}$, where $\rho_{n, s}$ is a random state with distribution $\mu_{n, s}$, and introduce the random variables $\alpha_{n, s}^{\prime}=\delta\left(\operatorname{sp}\left(\sqrt{n s} A_{n, s}\right), X_{n}^{\mathrm{sc}}\right)$ and $\beta_{n, s}^{\prime}=\delta\left(X_{n}^{\mathrm{sc}}, \operatorname{sp}\left(\sqrt{n s} A_{n, s}\right)\right)$. Since the distribution of $A_{n, s}$ is unitarily invariant, the same argument applies verbatim, and yields the inequalities

$$
\frac{1}{\sqrt{n s}} \phi_{K}\left(X_{n}^{\mathrm{sc}}\right) \mathbf{E}\left(\beta_{n, s}^{\prime}\right)^{-1} \leqslant \mathbf{E}\left\|\rho_{n, s}-\frac{\mathbb{I}}{n}\right\|_{K} \leqslant \frac{1}{\sqrt{n s}} \phi_{K}\left(X_{n}^{\mathrm{sc}}\right) \mathbf{E} \alpha_{n, s}^{\prime}
$$

By combining (27) and (28), one obtains the following inequalities for the constants $C_{n, s}$ and $c_{n, s}$ introduced in Proposition 3.1

$$
\frac{\mathbf{E}\left(\beta_{n, s}^{\prime}\right)^{-1}}{\mathbf{E} \alpha_{n}} \leqslant c_{n, s} \leqslant C_{n, s} \leqslant \frac{\mathbf{E} \alpha_{n, s}^{\prime}}{\mathbf{E} \beta_{n}^{-1}}
$$

It is now immediate to deduce Proposition 3.1 from the following lemma.

Lemma B.3. In the notation introduced above, we have

$$
\lim _{n \rightarrow \infty} \mathbf{E} \alpha_{n}=\lim _{n \rightarrow \infty} \mathbf{E} \beta_{n}^{-1}=\lim _{n, s / n \rightarrow \infty} \mathbf{E} \alpha_{n, s}^{\prime}=\lim _{n, s / n \rightarrow \infty} \mathbf{E}\left(\beta_{n, s}^{\prime}\right)^{-1}=1 .
$$

Proof of Lemma B.3. Propositions B.1 and B.2 combined with Proposition A.4 imply that $\alpha_{n}, \beta_{n}, \alpha_{n, s}^{\prime}$ and $\beta_{n, s}^{\prime}$ converge in probability to 1 when $n$ and $s / n$ tend to infinity.

The convergence of $\mathbf{E} \alpha_{n}$ will follow if we show that, say, $\sup _{n} \mathbf{E} \alpha_{n}^{2}<+\infty$. Introduce a vector $z \in \mathbf{R}^{n, 0}$ with $\lfloor n / 2\rfloor$ coordinates equal to 1 and $\lfloor n / 2\rfloor$ coordinates equal to -1 (if $n$ is odd, the remaining coordinate is necessarily 0). It is easily checked that $\delta\left(z, X_{n}^{\mathrm{sc}}\right)$ is bounded by an absolute constant $C$. Moreover, for any $x \in \mathbf{R}^{n, 0}$, we have $\delta(x, z)=\|x\|_{\infty}$. It follows that

$$
\alpha_{n} \leqslant C \frac{1}{\sqrt{n}}\left\|G_{n}\right\|_{\infty}
$$

and the problem is reduced to showing that $\sup _{n} \mathbf{E}\left\|G_{n} / \sqrt{n}\right\|_{\infty}^{2}<+\infty$, which follows easily from the tail estimates (26).

Since $\alpha_{n} \beta_{n} \geqslant 1$, convergence of $\mathbf{E} \beta_{n}^{-1}$ follows from the convergence of $\mathbf{E} \alpha_{n}$. The convergence of $\mathbf{E} \alpha_{n, s}^{\prime}$ and $\mathbf{E}\left(\beta_{n, s}^{\prime}\right)^{-1}$ is proved along the same lines. 
Remark B.4. The above arguments are quite flexible and become particularly simple if we do not aim at showing that $\delta(\cdot, \cdot) \sim 1$, but at an estimate $\delta(\cdot, \cdot) \lesssim 1$. For example, the estimates stated in the second remark following Proposition 3.1 can be deduced from the known bounds on $\mathbf{E}\|\cdot\|_{\infty}$ (such as (26)) for the ensembles in question and the following elementary fact: if $x, y \in \mathbf{R}^{n, 0}$ are such that $\max _{j}\left|x_{j}\right| \leqslant \frac{C}{n} \sum_{j}\left|y_{j}\right|$, then $x \prec 2 C y$.

\section{Appendix C. Irreducible subspaces for the isometry group of $\mathcal{S}$}

Fix an integer $d>1$. Denote by $H$ the (real) vector space $\mathcal{M}_{d}^{s a}, H_{0}=\mathcal{M}_{n}^{s a, 0}$ the hyperplane of trace zero matrices, and $H_{1}=H_{0}^{\perp}$ the one-dimensional space of scalar matrices. (Note that this notation is not identical to the one used in the main body of the paper.)

The unitary group $\mathcal{U}\left(\mathbf{C}^{d}\right)$ acts on $H$ by conjugacy: the action of an element $U \in \mathcal{U}\left(\mathbf{C}^{d}\right)$ is given by

$$
A \mapsto U A U^{\dagger} \text {. }
$$

Similarly, for any integer $k$, the direct product $\mathcal{U}\left(\mathbf{C}^{d}\right)^{k}$ acts on $H^{\otimes k}$ : the action of a $k$-tuple $\left(U_{1}, \ldots, U_{k}\right)$ being given by

$$
A \mapsto\left(U_{1} \otimes \cdots \otimes U_{k}\right) A\left(U_{1}^{\dagger} \otimes \cdots \otimes U_{k}^{\dagger}\right)
$$

(this construction is called external tensor product in representation theory).

When a group $\Gamma$ acts on a (real or complex) vector space $E$ (i.e., if $\mathcal{L}(E)$ is the space of linear operators on $E$ and $\pi: \Gamma \rightarrow \mathcal{L}(E)$ is a representation), one says that a nonzero subspace $F \subset E$ is invariant if, for every $\gamma \in \Gamma, \pi(\gamma) F \subset F$. We say that a nonzero subspace $F \subset E$ is irreducible if, for every nonzero $x \in F$,

$$
F=\operatorname{span}\{\pi(\gamma) x: \gamma \in \Gamma\}
$$

in other words, if $F$ is invariant, but no proper subspace of $F$ is.

Lemma C.1. Consider the action of $\mathcal{U}\left(\mathbf{C}^{d}\right)^{k}$ on $H^{\otimes k}$ given by (29). A subspace $E \subset H^{\otimes k}$ is irreducible if and only if it has the form

$$
H_{i_{1}} \otimes \cdots \otimes H_{i_{k}}
$$

for some choice of $\left(i_{1}, \ldots, i_{k}\right) \in\{0,1\}^{k}$. Moreover, a subspace $E \subset H^{\otimes k}$ is invariant if and only if it is the direct sum of subspaces of the form (30).

Before we prove Lemma C.1, let us state basic results from representation theory (see [42]).

Proposition C.2. The following results are valid for complex representations of compact groups.

(i) For every representation $\pi: \Gamma \rightarrow \mathcal{L}(E)$, there exists a decomposition of $E$ as direct sum

$$
E=\bigoplus_{\alpha} E_{\alpha}
$$

where $\left(E_{\alpha}\right)$ are irreducible subspaces.

(ii) If, moreover, there do not exist indices $\alpha \neq \alpha^{\prime}$ such that the subrepresentations of $\pi$ into $\mathcal{L}\left(E_{\alpha}\right)$ and $\mathcal{L}\left(E_{\alpha^{\prime}}\right)$ are isomorphic, then the decomposition in (31) is unique.

(iii) If $\pi_{1}: \Gamma_{1} \rightarrow \mathcal{L}\left(E_{1}\right)$ and $\pi_{2}: \Gamma_{2} \rightarrow \mathcal{L}\left(E_{2}\right)$ are two irreducible representations, then the (external) tensor product representation

$$
\pi_{1} \otimes \pi_{2}: \Gamma_{1} \times \Gamma_{2} \rightarrow \mathcal{L}\left(E_{1} \otimes E_{2}\right)
$$

is irreducible. 
The statements (i), (ii) and (iii) appear in [42] as Theorems 2, 8 and 10 respectively (note that although these theorems are stated in [42] for finite groups, they remain valid for compact groups, as noted in Chapter 4).

Proof of Lemma C.1. Let us switch to the complex field: denote by $E^{\mathbf{C}}$ the complexification of a real vector space $E$. Note that $H^{\mathbf{C}}$ naturally identifies with the space of complex $d \times d$ matrices, and $\left(H^{\otimes k}\right)^{\mathbf{C}}$ naturally identifies with $\left(H^{\mathbf{C}}\right)^{\otimes k}$.

We first check that the subspaces $H_{0}^{\mathbf{C}}$ and $H_{1}^{\mathbf{C}}$ are irreducible for the action of $\mathcal{U}\left(\mathbf{C}^{d}\right)$ on $H$. Obviously $H_{1}^{\mathbf{C}}$ is irreducible. Let $A \in H_{0}^{\mathbf{C}}$ with $A \neq 0$ and consider the (complex) space

$$
\mathcal{F}=\operatorname{span}\left\{U^{\dagger} A U: U \in \mathcal{U}\left(\mathbf{C}^{d}\right)\right\} .
$$

We must show that $\mathcal{F}=H_{0}^{\mathbf{C}}$. Note that $\mathcal{F}$ necessarily contains a matrix with the diagonal non identically zero (since one may diagonalize either the Hermitian or the anti-Hermitian part of $A$, and one of them is nonzero). Averaging over all diagonal unitary matrices shows then that $\mathcal{F}$ contains a nonzero diagonal matrix. Since the symmetric group $\mathfrak{S}_{d}$ acts irreducibly on the hyperplane of sum zero vectors in $\mathbf{C}^{d}$, we deduce that $A$ contains every diagonal trace zero matrix, and therefore every Hermitian or skew-Hermitian trace zero matrix, so that $\mathcal{F}=H_{0}^{\mathbf{C}}$.

By Proposition C.2(iii), it follows that for every $k \in \mathbf{N}$ and every $\left(i_{1}, \ldots, i_{k}\right) \in\{0,1\}^{k}$, the subspace

$$
E_{\left(i_{1}, \ldots, i_{k}\right)}=H_{i_{1}}^{\mathbf{C}} \otimes \cdots \otimes H_{i_{k}}^{\mathbf{C}}
$$

is an irreducible subspace in $\left(H^{\mathbf{C}}\right)^{\otimes k}$ for the action of $\mathcal{U}\left(\mathbf{C}^{d}\right)^{k}$. Moreover, the $2^{k}$ corresponding subrepresentations are pairwise non-isomorphic (indeed, for every $1 \leqslant j \leqslant k$, the index $i_{j}$ can be retrieved by looking at the subgroup corresponding to the $j$ th copy of $\mathcal{U}\left(\mathbf{C}^{d}\right)$ : its action on $E_{\left(i_{1}, \ldots, i_{k}\right)}$ is trivial iff $i_{j}=1$ ). By Proposition C.2(ii), this is the unique decomposition of $\left(H^{\mathbf{C}}\right)^{\otimes k}$ into irreducible subspaces, and any invariant subspace is obtained as the direct sum of some of these irreducible subspaces.

Note that a subspace $E \subset H^{\otimes k}$ is invariant if and only if its complexification $E^{\mathbf{C}} \subset\left(H^{\mathbf{C}}\right)^{\otimes k}$ is invariant. This implies the second part of Lemma C.1. The first part follows since the irreducible subspaces are minimal in the lattice of invariant subspaces.

\section{Appendix D. The $\ell$-Position}

Important: In this section we restrict our attention to symmetric convex bodies. While most of the concepts and results generalize to the non-symmetric case, it is not known whether the central result, Proposition D.4, holds in that setting.

Our presentation of $\ell$-position is standard and follows closely the book 38 . Let $K \subset \mathbf{R}^{m}$ be a symmetric convex body, and let $T: \mathbf{R}^{m} \rightarrow \mathbf{R}^{m}$ be a linear operator. We define the quantity $\ell_{K}(T)$ as

$$
\ell_{K}(T)=\mathbf{E}\|T(G)\|_{K},
$$

where $G$ denotes the standard Gaussian vector in $\mathbf{R}^{m}$ (i.e. the coordinates are independent $N(0,1)$ random variables). If there is no ambiguity about the underlying convex body, we write $\ell$ instead of $\ell_{K}$. The following proposition collects elementary properties of this concept.

Proposition D.1. If $K \subset \mathbf{R}^{m}$ is a symmetric convex body, then

(i) $\ell_{K}(\cdot)$ is a norm on $\mathcal{L}\left(\mathbf{R}^{m}\right)$,

(ii) $\ell_{K}\left(T_{1} T_{2}\right) \leqslant \ell\left(T_{1}\right)_{K}\left\|T_{2}\right\|_{o p}$ and $\ell_{K}\left(T_{1} T_{2}\right) \leqslant \ell_{K}\left(T_{2}\right)\left\|T_{1}\right\|_{o p}$ (this is called the ideal property),

(iii) $\ell_{K}(\mathbb{I})=w_{G}\left(K^{\circ}\right)=\gamma_{m} w\left(K^{\circ}\right) \sim \sqrt{m} w\left(K^{\circ}\right)$ and $\ell_{K}(u)=\ell_{u^{-1} K}(\mathbb{I})$, 
(iv) If $P_{E}$ denotes the orthogonal projection on a subspace $E \subset \mathbf{R}^{m}$, then

$$
\ell_{K}\left(P_{E}\right)=w_{G}\left((K \cap E)^{\circ}\right)=w_{G}\left(P_{E} K^{\circ}\right),
$$

where by $(K \cap E)^{\circ}$ we mean the polar inside $E$.

Before proceeding, let us point out that the more common definition of the $\ell$-norm is via the second moment, namely $\left(\mathbf{E}\|T(G)\|_{K}^{2}\right)^{1 / 2}$. However, the two expressions are equivalent.

Proposition D.2. For any symmetric convex body $K \subset \mathbf{R}^{m}$ and for any linear operator $T: \mathbf{R}^{m} \rightarrow \mathbf{R}^{m}$, we have

$$
\mathbf{E}\|T(G)\|_{K} \leqslant\left(\mathbf{E}\|T(G)\|_{K}^{2}\right)^{1 / 2} \leqslant \sqrt{\frac{\pi}{2}} \mathbf{E}\|T(G)\|_{K} .
$$

Proposition D.2 (which we do not really need here, but include for clarity) is a special case of Corollary 3 in [27]. If we do not insist on obtaining the optimal constant $\sqrt{\pi / 2}$, the result is more elementary (essentially a special case of the so-called Khinchine-Kahane inequality) and extends to non-symmetric convex bodies (see, e.g., 8], Lemma 3.3).

We now consider the maximization problem

$$
\max _{\ell(T) \leqslant 1} \operatorname{det} T .
$$

By compactness, the maximum is attained and it is obviously strictly positive. Since, for $V \in O(m)$ and for any $T$, we have $\ell(T)=\ell(T V)$, it follows that it is enough to restrict our attention to $T$ 's that are positive definite (PD). We claim that, under the PD restriction, the solution $T_{0}$ to (32) is unique. Indeed, if we had any other solution $T_{1}$, it would follow that $T=\left(T_{0}+T_{1}\right) / 2$ verifies, on the one hand, $\ell(T) \leqslant 1$ and, on the other hand, $\operatorname{det} T>\left(\operatorname{det} T_{0}\right)^{1 / 2}\left(\operatorname{det} T_{1}\right)^{1 / 2}=\operatorname{det} T_{0}($ by strict log-concavity of $\operatorname{det}$ over PD), a contradiction. Clearly, $\ell\left(T_{0}\right)=1$.

If the maximum above is attained when $T$ is a multiple of identity, one says that $K$ is in the $\ell$-position. For every symmetric convex body $K$, there is a linear transformation $T$ such that $T K$ is in the $\ell$-position; moreover $T$ is unique up to rotations and homotheties.

We will take advantage of the uniqueness of the $\ell$-position through the following lemma

Lemma D.3. Let $K \subset \mathbf{R}^{m}$ be a symmetric convex body and $\Gamma$ be the isometry group of $K$ (i.e. the set of orthogonal transformations $U$ such that $U K=K)$.

Then there is a linear map $T$ such that $T K$ is in the $\ell$-position and which has the form

$$
T=\sum_{i} \lambda_{i} P_{E_{i}}
$$

for some $\lambda_{i}>0$ and some subspaces $\left(E_{i}\right)$ which are invariant under the action of $\Gamma$.

Proof. Let $T \geqslant 0$ be the unique solution to the maximization problem (32). For any $\gamma \in \Gamma$ and $x \in \mathbf{R}^{m}$, we have $\|\gamma(T x)\|_{K}=\|T x\|_{K}$, hence $\ell(\gamma T)=\ell(T)$. Since $\ell(T V)=\ell(T)$ for any orthogonal transformation $V$, it follows that $\ell\left(\gamma T \gamma^{-1}\right)=\ell(T)$. Uniqueness of the solution implies that $\gamma T \gamma^{-1}=T$. Write

$$
T=\sum_{i} \lambda_{i} P_{i},
$$

where $\lambda_{i}>0$ are distinct positive numbers and $P_{i}$ pairwise orthogonal projectors. Given $i$, we have $\gamma P_{i} \gamma^{-1}=P_{i}$ for all $\gamma \in \Gamma$, in particular the range of $P_{i}$ is invariant under the action of $\Gamma$. 
We will use the following theorem which compares the mean width of $K$ and the mean width of $K^{\circ}$ whenever $K$ is a convex body in the $\ell$-position.

Proposition D.4. Let $K \subset \mathbf{R}^{m}$ be a symmetric convex body in the $\ell$-position. Then

$$
\ell_{K}(\mathbb{I}) \ell_{K^{\circ}}(\mathbb{I}) \lesssim m \log m
$$

or, equivalently,

$$
w(K) w\left(K^{\circ}\right) \lesssim \log m
$$

This deep result is known in the Asymptotic Geometric Analysis as the " $M M^{*}$-estimate". It follows by combining results of Figiel and Tomczak-Jaegermann with those of Pisier, including in particular sharp bounds on the so-called " $K$-convexity constant." See chapter 3 in 38] for a complete proof and references.

Incidentally, while $O(\log m)$ is the optimal general upper bound for the $K$-convexity constant mentioned above (see [11]), to the best of our knowledge it is not known whether it gives the correct order in Proposition D.4. The pair $\left(\ell_{1}^{m}, \ell_{\infty}^{m}\right)$ gives an example for which $w(K) w\left(K^{\circ}\right)$ is of order $\sqrt{\log m}$. In the non-symmetric case, the $m$-dimensional simplex $\Delta$ is an example with $w(\Delta) w\left(\Delta^{\circ}\right) \simeq \log m$, but known general upper bounds for non-symmetric convex bodies are much weaker [8, 40].

Note that the lower bound $w_{G}(K) w_{G}\left(K^{\circ}\right) \geqslant \gamma_{m}^{2} \sim m$ (or, equivalently, $w(K) w\left(K^{\circ}\right) \geqslant 1$ ) follows simply from

$$
\begin{aligned}
\gamma_{m} & =\mathbf{E}|G| \leqslant \mathbf{E}\left(\|G\|_{K}^{1 / 2}\|G\|_{K^{\circ}}^{1 / 2}\right) \leqslant\left(\mathbf{E}\|G\|_{K}\right)^{1 / 2}\left(\mathbf{E}\|G\|_{K^{\circ}}\right)^{1 / 2} \\
& =\gamma_{m} w\left(K^{\circ}\right)^{1 / 2} w(K)^{1 / 2} .
\end{aligned}
$$

Acknowledgements. Part of this research was performed during the Fall of 2010 while SJS and DY visited the Fields Institute in Toronto, Canada ("Thematic Program on Asymptotic Geometric Analysis"), and while GA and SJS visited Institut Mittag-Leffler ("Quantum Information Theory" programme). Thanks are due to these institutions and to their staff for their hospitality, and to fellow participants for many inspiring interactions. SJS thanks Harsh Mathur for providing insight into physicist's view of quantum theory.

The research of GA was supported in part by the Agence Nationale de la Recherche grants ANR-08-BLAN-031103 and ANR 2011-BS01-008-02.

The research of SJS was supported in part by grants from the National Science Foundation (U.S.A.), from the U.S.-Israel Binational Science Foundation, and by the second ANR grant listed under GA.

The research of DY was supported by the Fields Institute, the NSERC Discovery Accelerator Supplement Grant \#315830 (from Carleton University), and by ERA and NSERC discovery grants (from the University of Ottawa), while DY held a Fields-Ontario postdoctoral fellowship.

\section{REFERENCES}

[1] Anderson, G.; Guionnet, A.; Zeitouni, O. An Introduction to Random Matrices. Cambridge Studies in Advanced Mathematics, 118, 2009.

[2] Arveson, W. The probability of entanglement. Comm. Math. Phys. 286 (2009) 283-312.

[3] Aubrun, G. Partial transposition of random states and non-centered semicircular distributions. Random Matrices: Theory and Applications 1 (2012), no. 2, 1250001 (29 pages).

[4] Aubrun, G.; Szarek, S. Tensor products of convex sets and the volume of separable states on $N$ qudits. Phys. Rev. A 73 (2006) 022109.

[5] Aubrun, G.; Szarek, S.; Werner, E. Hastings's additivity counterexample via Dvoretzky's theorem. Comm. Math. Phys. 305 (2011) 85-97.

[6] Aubrun, G.; Szarek, S.; Ye, D. Phase transitions for random states and a semi-circle law for the partial transpose. Phy. Rev. A 85 (2012) 030302(R), 4 pages. 
[7] Bai, Z. D.; Yin, Y. Q. Convergence to the semicircle law. Ann. Probab. 16 (1988) 863-875.

[8] Banaszczyk, W.; Litvak, A.; Pajor, A.; Szarek, S. The flatness theorem for nonsymmetric convex bodies via the local theory of Banach spaces. Math. Oper. Res. 24 (1999) 728-750.

[9] Bengtsson, I.; Życzkowski, K. Geometry of Quantum States. Cambridge University Press, 2006.

[10] Bhatia, R. Matrix analysis. Graduate Texts in Mathematics, 169. Springer-Verlag, 1997.

[11] Bourgain, J. On Martingales Transforms in Finite Dimensional Lattices with an Appendix on the K-Convexity Constant. Math. Nachr. 119 (1984) 41-53.

[12] Collins, B.; Nechita, I.; Ye, D. The absolute positive partial transpose property for random induced states. Random Matrices: Theory and Applications 1 (2012) 1250002 (22 pages).

[13] Davidson, K. R.; Szarek, S. Local operator theory, random matrices and Banach spaces. In Handbook of the geometry of Banach spaces. Edited by W. B. Johnson and J. Lindenstrauss (North-Holland, Amsterdam, 2001), Vol. 1, pp. 317-366; (North-Holland, Amsterdam, 2003), Vol. 2, pp. 1819-1820.

[14] Einstein, A.; Podolsky, B.; Rosen, N. Can Quantum-Mechanical Description of Physical Reality Be Considered Complete? Phys. Rev. 47 (1935) 777-780.

[15] Fawzi, O.; Hayden, P.; Sen, P. From Low-Distortion Norm Embeddings to Explicit Uncertainty Relations and Efficient Information Locking. arXiv:1010.3007 v3 [quant-ph].

[16] Galambos, J. Advanced probability theory. Probability: Pure and Applied, 3. Marcel Dekker, Inc., New York, 1988.

[17] Gurvits, L. Classical deterministic complexity of Edmond's problem and quantum entanglement. Proceedings of the Thirty-Fifth Annual ACM Symposium on Theory of Computing, 10-19 (electronic), ACM, New York, 2003.

[18] Gurvits, L.; Barnum, H. Largest separable balls around the maximally mixed bipartite quantum state. Phys. Rev. A 66 (2002) 062311.

[19] Gurvits, L.; Barnum, H. Better bound on the exponent of the radius of the multipartite separable ball. Phys. Rev. A $72(2005) 032322$.

[20] Haagerup, U.; Thorbjørnsen, S. Random matrices with complex Gaussian entries. Expositiones Math. 21 (2003) $293-337$.

[21] Hastings, M. B. Superadditivity of communication capacity using entangled inputs. Nature Physics 5 (2009) $255-257$.

[22] Hayden, P.; Leung, D.; Winter, A. Aspects of generic entanglement. Comm. Math. Phys. 265 (2006) 95-117.

[23] Horodecki, P. Separability criterion and inseparable mixed states with positive partial transposition. Phys. Lett. A 232 (1997) 333-339.

[24] Horodecki, M.; Horodecki, P.; Horodecki, R. Separability of mixed states: necessary and sufficient conditions. Phys. Lett. A 223 (1996) 1-8.

[25] Horodecki, M.; Horodecki, P.; Horodecki, R. Mixed-state entanglement and distillation: Is there a "bound" entanglement in nature? Phys. Rev. Lett. 80 (1998) 5239-5242.

[26] Kendon, V. M.; Życzkowski, K.; Munro, W. J. Bounds on entanglement in qudit subsystems. Phys. Rev. A 66 (2002) 062310 .

[27] Latała, R.; Oleszkiewicz, K. Gaussian measures of dilatations of convex symmetric sets. Ann. Probab. 27 (1999) 19221938.

[28] Ledoux, M. The concentration of measure phenomenon. Mathematical Surveys and Monographs, 89. American Mathematical Society, Providence, RI, 2001.

[29] Lévy, P. Problémes concrets d'analyse fonctionnelle. 2nd ed. Gauthier-Villars, Paris, 1951.

[30] Li, W. V.; Shao, Q. M. Gaussian processes: Inequalities, small ball probabilities and applications. In Stochastic processes: theory and methods. D.N. Shanbhag and C.R. Rao, Editors. Handbook of Statist. 19, 533-597, Elsevier 2001.

[31] Marchenko, V. A.; Pastur, L. A. The distribution of eigenvalues in certain sets of random matrices. Mat. Sb. 72 (1967) 507-536.

[32] Mehta, M. L. Random matrices. Academic Press, 2nd edition 1990.

[33] Milman, V. D.; Pajor, A. Entropy and Asymptotic Geometry of Non-Symmetric Convex Bodies. Adv. Math. 152 (2000) 314-335.

[34] Milman, V. D.; Schechtman, G. Asymptotic theory of finite dimensional normed spaces. With an appendix by M. Gromov, Lecture Notes Math. 1200, Springer Verlag, Berlin-New York, 1986.

[35] Mini-Workshop: Geometry of Quantum Entanglement. Abstracts from the mini-workshop held December 6-12, 2009. Organized by A. Buchleitner, S. Szarek, E. Werner and K. Życzkowski. Reporter: G. Aubrun. Oberwolfach Rep. 6, no. 4, 2993-3031, EMS, Zürich, 2009.

[36] Nielsen, M. A.; Chuang, I. L. Quantum Computation and Quantum Information. Cambridge University Press, 2000. 
[37] Peres, A. Separability Criterion for Density Matrices. Phys. Rev. Lett. 77 (1996) 1413-1415.

[38] Pisier, G. The volume of convex bodies and Banach space geometry. Cambridge Tracts in Mathematics, 94. Cambridge University Press, 1989.

[39] Rogers, C. A.; Shephard, G. C. Convex bodies associated with a given convex body. J. London Math. Soc. 33 (1958) $270-281$.

[40] Rudelson, M. Distances Between Non-symmetric Convex Bodies and the $M M^{*}$-estimate. Positivity 4 (2000) 161-178.

[41] Ruskai, M. B.; Werner, E. M. Bipartite states of low rank are almost surely entangled. J. Phys. A: Math. Theor. 42 (2009) 095303.

[42] Serre, J.-P. Linear representations of finite groups. Graduate Texts in Mathematics, Vol. 42. Springer-Verlag, New York-Heidelberg, 1977.

[43] Silverstein, J. W. The smallest eigenvalue of a large dimensional Wishart matrix. Ann. Probab. 13 (1985) $1364-1368$.

[44] Shor, P. W. Algorithms for quantum computation: discrete logarithms and factoring. IEEE Symposium on Foundations of Computer Science (1994) 124-134.

[45] Størmer, E. Positive linear maps of operator algebras. Acta Math. 110 (1963) 233-278.

[46] Szarek, S. The volume of separable states is super-doubly-exponentially small in the number of qubits. Phys. Rev. A 72 (2005) 032304.

[47] Szarek, S.; Bengtsson, I.; Życzkowski, K. On the structure of the body of states with positive partial transpose. J. Phys. A: Math. Gen. 39 (2006) L119-L126.

[48] Szarek, S.; Werner, E.; Życzkowski, K. Geometry of sets of quantum maps: a generic positive map acting on a highdimensional system is not completely positive. J. Math. Phys. 49 (2008) 032113.

[49] Szarek, S.; Werner, E.; Życzkowski, K. How often is a random quantum state k-entangled? J. Phys. A: Math. Theor. 44 (2011) 045303.

[50] Walgate, J.; Scott, A. J. Generic local distinguishability and completely entangled subspaces. J. Phys. A: Math. Theor. $41(2008) 375305$.

[51] Werner, R. F. Quantum states with Einstein-Podolsky-Rosen correlations admitting a hidden-variable model. Phys. Rev. A 40 (1989) 4277-4281.

[52] Woronowicz, S. L. Positive maps of low dimensional matrix algebra. Rep. Math. Phys. 10 (1976) $165-183$.

[53] Ye, D. On the Bures volume of separable quantum states. J. Math. Phy. 50 (2009) 083502.

[54] Ye, D. On the comparison of volumes of quantum states. J. Phys. A: Math. Theor. 43 (2010) 315301 (17pp).

[55] Życzkowski, K.; Sommers, H.-J. Hilbert-Schmidt volume of the set of mixed quantum states. J. Phys. A 36 (2003) 10115-10130.

[56] Życzkowski, K.; Sommers, H.-J. Induced measures in the space of mixed quantum states. J. Phys. A 34 (2001) 7111.

Guillaume Aubrun, Institut Camille Jordan, Université Claude Bernard Lyon 1, 43 Boulevard du 11 Novembre 1918, 69622 Villeurbanne CEDEX, France.

E-mail: aubrun@math.univ-lyon1.fr

Stanisław Szarek, Case Western Reserve University, Cleveland, Ohio 44106-7058, USA and Institut de Mathématiques de Jussieu, Université Pierre et Marie Curie, 75005 Paris, France

Email: szarek@cwru.edu

Deping Ye, School of Mathematics and Statistics, Carleton University, Ottawa, ON, K1S5B6, Canada, and The Fields Institute, Toronto, ON, M5T3J1, Canada.

Current address: Department of Mathematics and Statistics, Memorial University of Newfoundland, St. John's, Newfoundland, Canada A1C $5 S 7$

Email: deping.ye@mun.ca 